CrossMark <click for updates

Cite this: Phys. Chem. Chem. Phys., $2016,18,6351$

Received 22nd June 2015 Accepted 19th August 2015

DOI: $10.1039 / c 5 c p 03599 f$

www.rsc.org/pccp

\section{Modelling of graphene functionalization}

\author{
Martin Pykal, Petr Jurečka, František Karlický and Michal Otyepka*
}

Graphene has attracted great interest because of its remarkable properties and numerous potential applications. A comprehensive understanding of its structural and dynamic properties and those of its derivatives will be required to enable the design and optimization of sophisticated new nanodevices. While it is challenging to perform experimental studies on nanoscale systems at the atomistic level, this is the 'native' scale of computational chemistry. Consequently, computational methods are increasingly being used to complement experimental research in many areas of chemistry and nanotechnology. However, it is difficult for non-experts to get to grips with the plethora of computational tools that are available and their areas of application. This perspective briefly describes the available theoretical methods and models for simulating graphene functionalization based on quantum and classical mechanics. The benefits and drawbacks of the individual methods are discussed, and we provide numerous examples showing how computational methods have provided new insights into the physical and chemical features of complex systems including graphene and graphene derivatives. We believe that this overview will help non-expert readers to understand this field and its great potential.

\section{Introduction}

Graphene $^{1}$ is a two dimensional material consisting of a hexagonal (honeycomb) lattice of covalently bound $\mathrm{sp}^{2}$ carbon atoms that are sandwiched between two $\pi$-electron clouds. Despite extensive research efforts triggered by numerous potential applications

Regional Centre of Advanced Technologies and Materials, Department of Physical Chemistry, Faculty of Science, Palacký University Olomouc, tř. 17. listopadu 12, 77146 Olomouc, Czech Republic. E-mail: Michal.Otyepka@upol.cz; Fax: +420 585634 761; Tel: +420 585634756

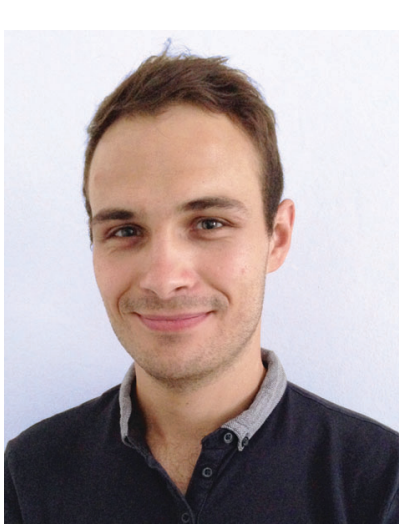

Martin Pykal
Martin Pykal is a PhD student at the Palacký University, Olomouc. His main areas of interest are molecular dynamics simulations of graphene and other carbon nanostructures and their interactions with small biomolecules.

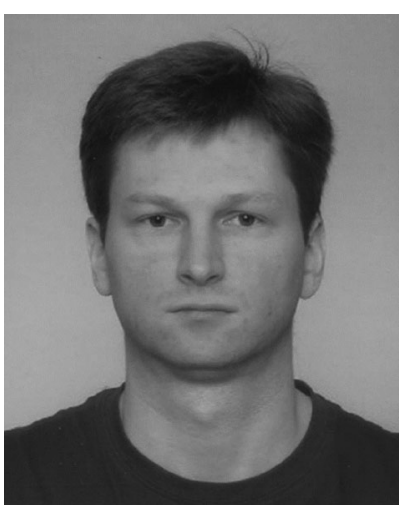

Petr Jurečka of graphene and its derivatives (Fig. 1), ${ }^{2}$ only a limited number of graphene-based products have been successfully commercialized to date. The graphene-based technology is still mainly in the research and development stage (for a more detailed discussion, please see the October 2014 issue of Nature Nanotechnology ${ }^{3}$ ). Among other purposes, it has diverse uses in sensing, ranging from the detection of small molecules ${ }^{4}$ to large biomacromolecules, ${ }^{5,6}$ including also DNA translocation ${ }^{7}$ and selective molecular sieving. ${ }^{8}$ The potential range of applications for graphene can be enhanced enormously by covalent and non-covalent modification. ${ }^{9}$ Covalent modification entails the formation of chemical bonds between graphene and nucleic acids, and intermolecular interactions in nanomaterials. 


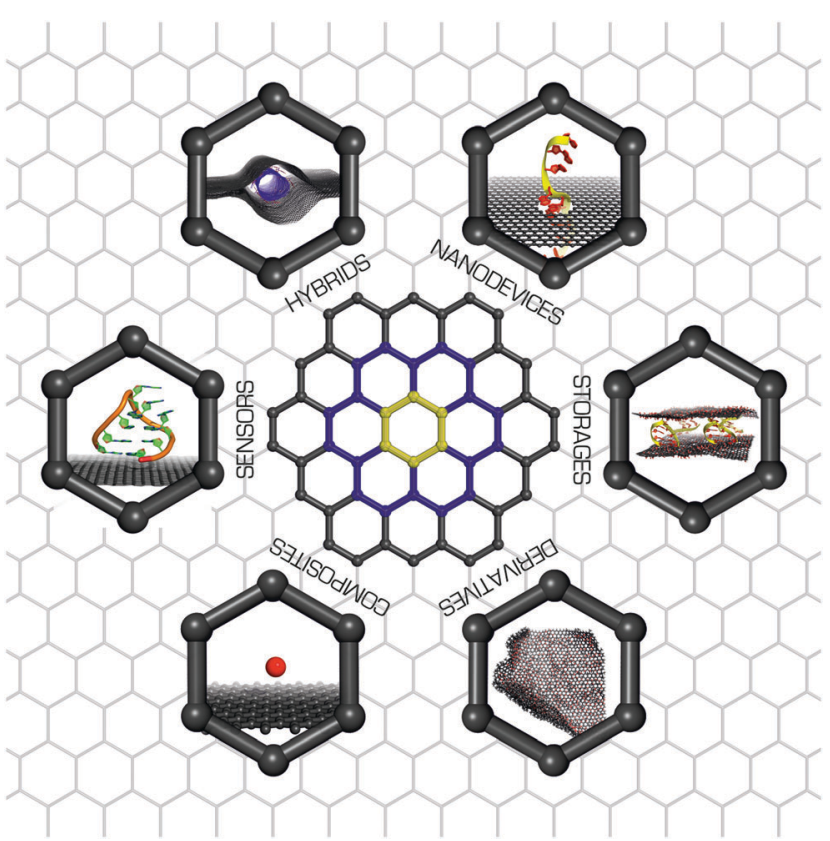

Fig. 1 Areas where graphene and its derivatives may have valuable applications.

some modifiers, which significantly change the structure and the hybridization of its carbon atoms. Such changes have profound effects on the material's physicochemical properties. ${ }^{10}$ Conversely, non-covalent modification entails the adsorption of a modifier onto the graphene surface via weak non-covalent forces. Such adsorption also changes the structure and properties, but to a lesser degree than the covalent modification; the magnitude of the changes is proportional to the modifier's binding energy. It should however be noted that the transition between covalent and non-covalent modification is rather smooth. To understand the effects of these modifications, and their behaviour in sensing applications, it is necessary to obtain an in-depth understanding of the nature and strength of the interactions between graphene and guest molecules. Computational chemistry is a valuable source of information that can be used to develop such an understanding.

Modelling of the interactions between graphene and guest molecules or modifiers can provide important insights into the effects of graphene modifications. This can be achieved using either electronic structure methods based on quantum mechanics, which explicitly account for the electronic structure of the studied molecular systems, or with molecular mechanics methods (also known as empirical force fields) that simplify molecular systems by representing them as collections of covalently bound van der Waals spheres. This perspective provides an overview of electronic structure and empirical methods (Sections 3 and 4) that can be used in computational studies on graphene modifications, extended with basic simulation methods for nuclear degrees of freedom (Section 5). We also provide some guidance for non-experts to explain which methods are applicable in particular contexts and how suitable they are for predicting the behaviour and properties of functionalized graphene and graphene derivatives. Finally we present numerous illustrative examples of computational studies that have enhanced our understanding of modified graphene (Section 6).

\section{Graphene models}

\subsection{Finite molecular models of graphene}

Graphene is often modelled as a finite polyaromatic hydrocarbon $(\mathrm{PAH})^{11-14}$ such as coronene $\left(\mathrm{C}_{24} \mathrm{H}_{12}\right)$ or circumcoronene $\left(\mathrm{C}_{54} \mathrm{H}_{18}\right)$, both of which are shown in Fig. $2 .{ }^{15,16}$ The carbon networks of these model molecules are capped with hydrogen atoms that saturate the dangling bonds at their edges. This affects the distribution of electronic density within the system because the electrons of the hydrogens are drawn to the carbon skeleton, generating a positive electrostatic potential on the

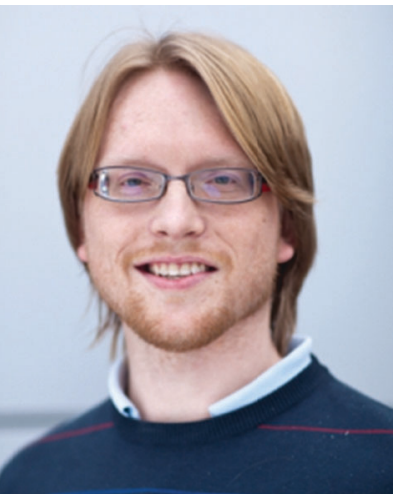

František Karlický

František Karlický graduated in 2004 at the University of Ostrava, Czech Republic. He obtained a PhD degree in physical chemistry from the Prague Institute of Chemical Technology in 2009 and his PhD work was focused on the development of methods for solving the Schrödinger equation for many-body bosonic systems. He is now an assistant professor at the Department of Physical Chemistry and a junior

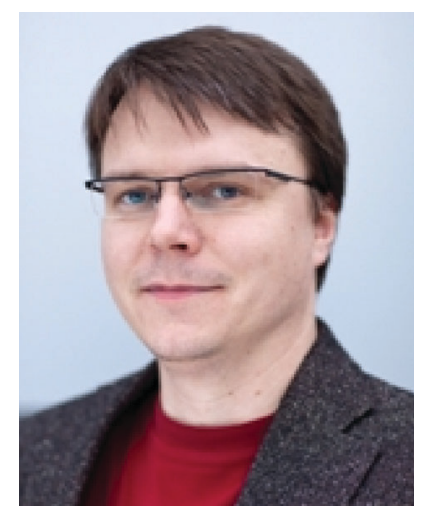

Michal Otyepka
Michal Otyepka received his $P h D$ degree in physical chemistry at the Palacky University, Olomouc (2004). Currently, he is a head of the Department of Physical Chemistry and vice-director of the Regional Centre of Advanced Technologies and Materials, both at the Palacky University, Olomouc, Czech Republic. His research is mostly focused on modelling of biomacromolecules, $2 D$ materials and their interactions, and chemistry of graphene derivatives. In 2014, he received Impulse from Neuron fund to support science.

researcher at the Regional Centre of Advanced Technologies and Materials, Palacký University, Olomouc. His research interests include modelling of carbon nanostructures, transition metal complexes and atomic clusters. 
A)

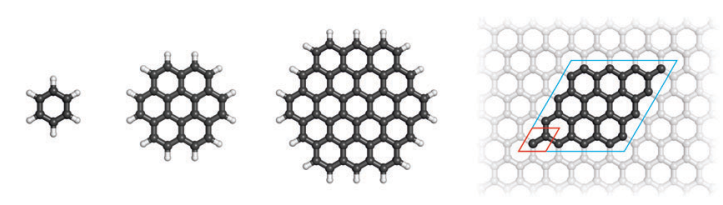

B)
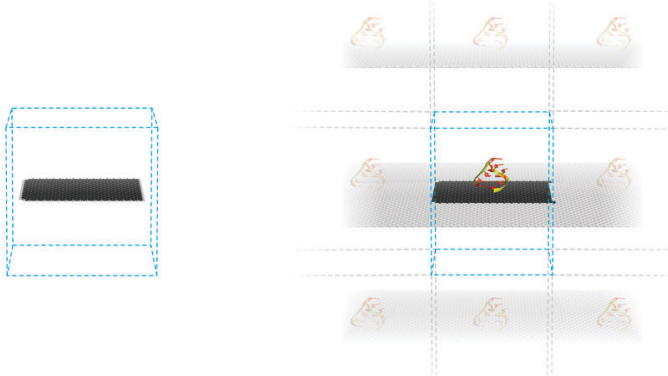

Fig. 2 (A) Some aromatic hydrocarbons that are commonly used as nonperiodic models of graphene in quantum calculations (benzene, coronene and circumcoronene), and a supercell of 32 carbon atoms from a periodic graphene model, with a unit cell highlighted in red. (B) Simulation boxes for empirical models containing a finite graphene flake (left) and a periodic graphene sheet with a small adsorbed RNA molecule (right). In techniques based on periodic boundary conditions, the supercell/simulation box is replicated throughout the space.

hydrogen atoms and a negative electrostatic potential above and below the carbon sheet where the $\pi$ electron cloud is located. Consequently, polyaromatic hydrocarbons have significant quadrupole moments that depend on their size (Fig. 3). ${ }^{17}$ This finite quadrupolar potential means that PAHs are imperfect models for the infinite flat periodic sheet of graphene, in which the quadrupolar potential completely vanishes. It should be noted, however, that real graphene is corrugated and the quadrupolar potential may be nonzero near its surface (Fig. 3). An important advantage of using finite molecular models is that they can be studied using a wide portfolio of electronic structure

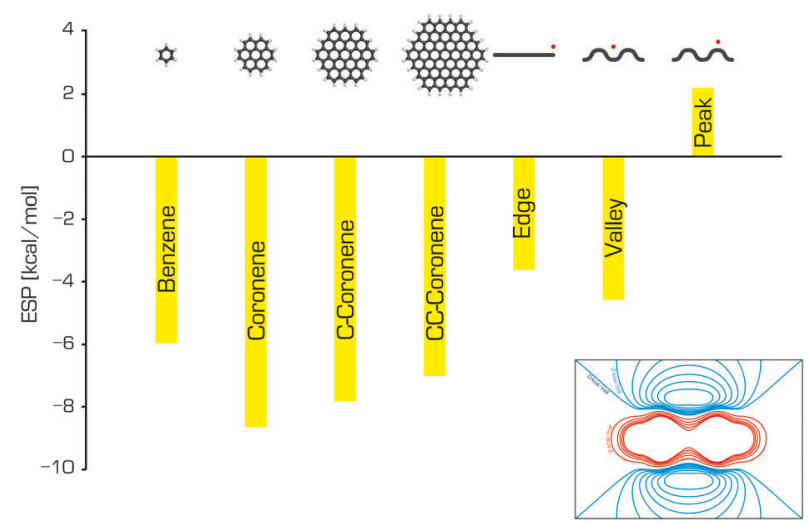

Fig. 3 The electrostatic potentials of benzene, coronene, circumcoronene and circumcircumcoronene (calculated in the middle of the molecule, $3.4 \AA$ above the surface), and the electrostatic potentials at specific positions relative to a graphene sheet (adapted from ref. 17). The inset shows the ESP around the benzene molecule; the red and blue contours represent positive and negative potentials, respectively. methods developed for molecular systems. The only limitations come from the size of the system that can be treated in a reasonable timeframe with specific methods, and the available computational power. A systematic study by Hobza and coworkers showed that the interaction energies of tetracyanoethylene and tetracyanoquinodimethane with various PAHs decreased convergently as the size of PAH increased. ${ }^{18}$

Empirical methods (Section 4) allow the use of substantially larger models of graphene flakes (up to tens of nanometers), and are therefore applicable when studying nanoscale phenomena such as exfoliation and aggregation processes in colloidal dispersions of graphene. ${ }^{19}$ In such cases edge effects as well as the effects of the system's quadrupole moment may become important. ${ }^{17}$ Shih and coworkers ${ }^{20}$ studied the stability and mechanisms of the aggregation process in exfoliated graphene solutions in several frequently used polar solvents. Based on their simulations and kinetic theory, they proposed a model of graphene aggregation in which the dominant barrier to aggregation was associated with the energetic cost of eliminating a single layer of solvent molecules confined between two graphene sheets oriented in parallel. Colloidal dispersions of graphene were also investigated by Lin et al., ${ }^{21}$ who examined the morphology and kinetics of self-assembled structures of surfactants and graphene sheets. Their findings suggest that the surfactant molecules stabilized the colloidal graphene dispersion and prevented the re-formation of new two- and three-layered graphene aggregates. Freestanding graphene was also considered in a study on wrinkles on the graphene surface and their effect on the specific surface area. ${ }^{22}$ The results indicated that wrinkles could only change the specific surface area by $2 \%$ at most, regardless of their shape, the nature of the defects that were present, or the strain acting on the area.

\subsection{Periodic graphene}

Ideal graphene is an infinite two-dimensional (2D) sheet with a regular lattice structure. Such a material can be straightforwardly modelled using periodic boundary conditions (Fig. 2) in which a unit cell including two carbon atoms is replicated across space. This periodic graphene model can be studied using numerous methods, most of which are based on density functional theory (DFT) and were developed by solidstate physicists to model the physical features of crystals. When studying the adsorption of guest molecules (adsorbates) to graphene, the size of a replicating cell, which is known as the supercell, is dictated by the size and target concentration of the adsorbate because it is important to avoid unwanted interactions between replicas. Since the periodic boundary conditions are typically implemented over the three-dimensional (3D) space, graphene (which is generally assumed to lie in the $x y$ plane) and its complexes are modelled using 3D unit cells with a large vertical length $(\sim 1.5 \mathrm{~nm})$ to avoid spurious vertical interactions between replicas. Spurious interactions could be particularly problematic if the supercell contains polar molecules or ions, because of the slow decay of Coulombic forces. It should be noted that the attractive van der Waals (vdW) forces 
in nanomaterials act over longer distances than was originally assumed. ${ }^{23}$

Periodic models can also be used with empirical methods (Section 4). One of their advantages is that they help to avoid some artefacts that can be caused by the presence of edges. An example is the quadrupole moment, which should be considered when working with finite graphene models such as those discussed above. The electronic band structure of graphene and its derivatives can only reasonably be studied using periodic models because models that do not account the inherent extended nature of graphene neglect correlation contributions from the bands close to the Dirac point. Furthermore, the infinite model may better describe the situations encountered in some experiments, such as those involving measurements on spots of graphene flakes that may be multiple micrometers in diameter. In such cases, the presence of edge effects in a simulated finite sheet could introduce undesirable bias. An infinite periodic boundary condition (PBC) model was used to study the mechanism by which graphene dispersions are stabilized in the presence of lipids, revealing that the lipids present a kinetic barrier to graphene aggregation by forming reverse micelles on the graphene surface. ${ }^{24}$ On the other hand, PBC models may be less suitable for studying phenomena such as surface corrugation because the box size limits the scale on which corrugation effects can be studied. Another potential drawback of the periodic model that may be encountered with certain simulation configurations relates to sandwiched structures in which two graphene sheets are separated by a fixed distance; this can lead to unphysical conditions such as unreasonable pressures. It should also be noted that not every software package for performing empirical computations supports periodic models.

As mentioned above, both finite and infinite (periodic) graphene models can be described using either quantum chemical (electronic structure) or molecular mechanical (empirical) methods. The potential applications of each are delineated by the BornOppenheimer approximation, which enables the separation of electronic and nuclear motions inside a molecular system. Phenomena involving changes in electronic states should be modelled using electronic structure methods that explicitly account for electronic motions. Molecular mechanics can be used to model phenomena in which the electronic structure does not change or changes only slightly, such as changes in conformational states or physisorption.

\section{Electronic structure methods}

\subsection{Methods for studying non-covalent complexes of graphene}

We have already mentioned that graphene can be modified either covalently or non-covalently. However, the mode of adsorbate binding may in reality lie somewhere between these two extremes. To model such situations it is necessary to use theoretical methods that accurately describe both covalent and non-covalent forces. It should be stressed that the accurate description of non-covalent forces is quite challenging for current theoretical methods. To avoid lengthy descriptions of the many electronic structure methods that could potentially be used to describe the electronic and physical-chemical properties of graphene, we will focus here on methods that can be used to predict its non-covalent interactions with reasonable confidence. The fidelity of theoretical methods for chemical modifications of graphene will be discussed only with reference to specific cases. It is generally accepted that individual sheets of graphene are bound by London dispersion forces in graphite. London forces originate from non-local electron correlation effects. ${ }^{25}$ Any electronic structure theory must therefore account properly for these non-local correlation effects in order to reliably predict the properties of non-covalent graphene complexes such as their binding energies and geometries.

\subsection{Wavefunction based methods}

The Hartree-Fock (HF) method fails to describe electron correlation effects because it neglects the correlation between electrons of opposite spin. It is therefore necessary to use post-HF methods to address this deficiency. The second-order MøllerPlesset perturbation method (MP2) accounts for a large fraction of the electron correlation effect, but it has some drawbacks. First, it is significantly more computationally demanding than the HF method and tends to overestimate the binding energies of non-covalent complexes that are bound mostly by London dispersive forces. Several methods that derive from MP2 but offer greater accuracy have been developed. The spin-component scaled MP2 (SCS-MP2) ${ }^{26}$ and SCS(MI)-MP2 ${ }^{27}$ methods are of particular note because they predict binding energies significantly more accurately than MP2 without any additional computational cost. The CCSD method itself is not suitable for the accurate description of dispersion bonded complexes. However, its spin-component scaled variants SCS-CCSD $^{28}$ and SCS(MI)-CCSD, the latter of which is optimized for the study of molecular interactions, ${ }^{29}$ provide remarkably accurate results with a very good accuracy/computational cost ratio. The scaled MP2/MP3 method including higher-order correlation effects (e.g., MP2.5 $)^{30}$ can also be useful for obtaining very accurate binding energies for non-covalent complexes at an affordable computational cost. The current gold standard for predicting the binding energies of non-covalent complexes is undoubtedly the coupled cluster method including single, double and perturbative triple excitations - CCSD(T). Unfortunately, $\operatorname{CSSD}(\mathrm{T})$ calculations are so computationally demanding that only small systems of less than $\sim 35$ atoms can be studied in this way (Table 1 ). Significant speedups of CCSD and $\operatorname{CCSD}(\mathrm{T})$ calculations have been achieved using the recently-introduced domain based local pair-natural orbital (DLPNO) approximation, yielding the modified DLPNO-CCSD ${ }^{31}$ and DLPNO-CCSD(T $)^{32}$ methods. However, further testing of these methods may be required before they can be considered suitable for routine use. More detailed information on the performance of various methods for modelling non-covalent complexes can be found in a recent review. ${ }^{33}$

Wavefunction-based methods are always used in conjunction with a finite basis set. In the literature, combinations of a 
Table 1 Overview of electronic structure methods (see the text for abbreviations) that can be used to study complexes of graphene. Methods applicable to finite and periodic models are indicated with an " $\times$ ". For each method, the size of the model (in terms of its number of atoms) that can be treated, the computational cost, and the quality of the results obtained are indicated by sets of asterisks, with one asterisk indicating small models/low computational costs/good quality results, and four asterisks indicating large systems/huge costs/best quality results

\begin{tabular}{|c|c|c|c|c|c|}
\hline Method & Finite & $\mathrm{PBC}$ & Size & Cost & Quali \\
\hline \multicolumn{6}{|l|}{ WFT } \\
\hline MP2 & $x$ & $x$ & ** & ** & * \\
\hline SCS(MI)-MP2 & $x$ & $x$ & ** & ** & ** \\
\hline MP2.5 & $x$ & - & ** & *** & *** \\
\hline $\operatorname{CCSD}(\mathrm{T})$ & $\times$ & - & * & $* * * *$ & $* * * *$ \\
\hline \multicolumn{6}{|l|}{ DFT } \\
\hline M06-2X & $x$ & $x$ & *** & ** & ** \\
\hline DFT-D2, DFT-D3 ${ }^{a}$ & $x$ & $\times$ & *** & ** & ** \\
\hline DFT-TS $^{a}$ & $x$ & $x$ & *** & ** & ** \\
\hline vdW-DF, vdW-DF2 & $x$ & $x$ & *** & *** & ** \\
\hline optB88-vdW & $x$ & $x$ & *** & *** & ** \\
\hline RPA & $x$ & $x$ & * & $* * * *$ & $* * *$ \\
\hline \multicolumn{6}{|l|}{ Other } \\
\hline QMC & $x$ & $x$ & ** & $* * * *$ & ***** \\
\hline PM6-DH, SCC-DFTB-D & $x$ & $x$ & $* * * *$ & * & * \\
\hline
\end{tabular}

${ }^{a}$ The real performance and cost of DFT-D2, -D3, and -TS methods are determined by the underlying functional; hybrids are more expensive than GGA functionals.

method and a basis set are typically denoted in the form of a method/basis set - for example, SCS(MI)-MP2/cc-pVTZ, where cc-pVTZ stands for the correlation consistent polarized valence triple-zeta basis set developed by Dunning and coworkers. ${ }^{34}$ Many different basis sets have been developed, and a detailed description of their construction and applicability would be beyond the scope of this review; the interested reader can find more detailed information elsewhere. ${ }^{35}$ However, it should be noted that the chosen basis set can significantly affect the quality of the results obtained in any quantum chemical calculation. It is generally accepted that larger basis sets provide better results. This idea resulted in the development of extrapolation schemes, ${ }^{36-38}$ which estimate the results for an infinite basis set that is referred to as the complete basis set (CBS). Calculations performed at the $\operatorname{CCSD}(\mathrm{T}) / \mathrm{CBS}$ level of theory provide very accurate estimates for quantities such as the interaction energies of non-covalent complexes. ${ }^{33,39,40}$ When CBS extrapolation cannot be performed and small or medium size basis sets are used, which is usually the case, it is important to apply a correction for the basis set superposition error (BSSE) (Fig. 4) such as the counterpoise (CP) correction of Boys and Bernardi. ${ }^{41}$ The BSSE arises from the fact that the basis sets used to describe non-covalent complexes are necessarily larger than those used for their individual components (in the simple case of a dimeric complex, the basis set for the dimer will necessarily be twice the size of that for the separated monomer). Failure to correct the BSSE inevitably leads to an overestimation of binding energies. However, the $\mathrm{CP}$ correction is imperfect and frequently overestimates the $\mathrm{BSSE}^{42}$ so some authors use either the fractional BSSE correction or combine the CP with special extrapolation schemes. ${ }^{38,43}$
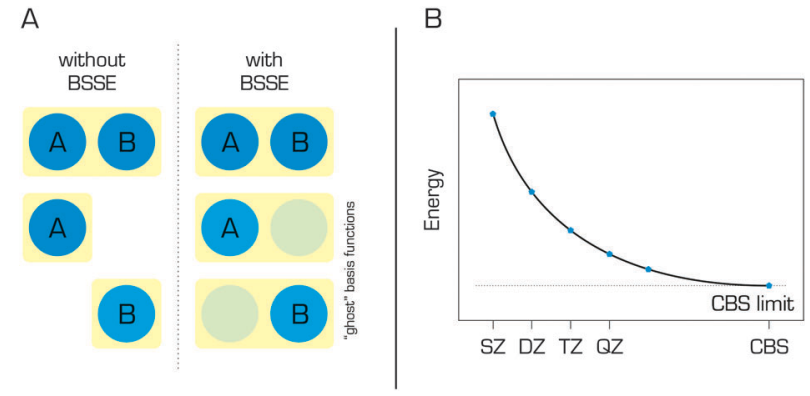

Fig. 4 (A) The interaction energy of two atoms or molecules is typically calculated as the energy difference between the complex $(A+B)$ and its components ( $A$ and $B$ ). In the counterpoise correction, the energy of each subsystem is calculated in the basis set of the whole complex, using "ghost" basis functions located at the original positions of the atomic centres of the other subsystem without the associated charges and electrons. (B) The convergence of energy with increasing basis set size (i.e. going from the minimal single-zeta (SZ) basis set to the double-(DZ), triple-(TZ) and quadruple zeta (QZ) sets) can be used to extrapolate the energy at the complete basis set (CBS) limit.

The post-HF methods were primarily developed for the study of molecular systems and they are readily applied to molecules and their assemblies. On the other hand, their applicability under periodic boundary conditions is currently very limited. ${ }^{44}$ The MP2 method has been implemented in a way that is compatible with the periodic boundary approach ${ }^{45-48}$ but calculations using this implementation are impractical for graphene because of its zero band gap. The CCSD method has been implemented in the VASP code for periodic boundary simulations $^{49}$ but this update has not yet been released to the public.

\subsection{Density functional methods}

Classical DFT methods based on the local density approximation (LDA), the generalized gradient approximation (GGA), or hybrid functionals do not account for non-local electron correlation effects, which are critical for the correct description of London dispersion forces. ${ }^{50-52}$ In LDA the binding caused by a too strong exchange contribution to the exchange-correlation functional is very different from the dynamical correlation effects promoting dispersion interactions. Several strategies have been developed to describe London dispersion forces within the framework of DFT. These include the empirically corrected DFT methods (abbreviated as DFT-D). The first DFT-D methods were based on summation over pair-wise $c_{i j} / r_{i j}{ }^{6}$ terms (where $c_{i j}$ represents an empirical dispersion coefficient for the electron pair $i j$ at a distance of $r_{i j}$ ), which were multiplied by a damping function (whose parameterization critically influences the accuracy of DFT-D) to avoid double counting of dispersive contributions,${ }^{53,54}$ which is necessary because DFT natively accounts for local electron-electron correlation. After the initial success of the DFT-D method ${ }^{53,55}$ a series of more sophisticated methods with better performance were introduced including DFT-D2 ${ }^{56}$ DFT-D $3{ }^{57}$ and DFT-TS. ${ }^{58}$ In addition, it was shown that many-body dispersion methods that go beyond pair-wise $\mathrm{vdW}$ interactions are required to improve the description of 
non-covalent interactions involving graphene. ${ }^{59,60}$ Dispersion can also be accounted for by combining DFT and MP2 calculations, the latter of which naturally account for long-range correlation. ${ }^{61}$ Such methods are called double hybrids because they include some portion of HF exchange in addition to the MP2 correlation. Double hybrid methods can be very accurate. $^{62,63}$ However, like MP2 calculations, they cannot be applied to periodic graphene. Note also that double hybrids somewhat underestimate long-range dispersion, although this can be corrected for by introducing empirical dispersion correction terms. ${ }^{64}$

An alternative strategy resulted in the development of non-local density functionals that account directly for dispersive correlation effects. Approaches of this type include the vdW-DF method of Dion et al.,${ }^{65}$ its improved successor vdW-DF2 $2{ }^{66}$ the reparameterized version optB88-vdW, ${ }^{67}$ and the VV10 method of Vydrov $e t$ al. ${ }^{68} \mathrm{It}$ should be noted that functionals which account for electron-electron correlation effects can be systematically improved by exploiting the adiabatic connection fluctuationdissipation theorem ${ }^{69}$ as clearly explained by Tkatchenko. ${ }^{70}$ Yet another way of modelling mid-range intermolecular interactions accurately with DFT is to use one of the highly parameterized local, GGA or meta-GGA DFT functionals developed by Truhlar and coworkers, which are called the Minnesota functionals (e.g. M06-2 $\left.\mathrm{X}^{71}\right)$. These functionals provide surprisingly good results at affordable cost (The comparison with other methods is shown in Table 1). The ability of some of these methods to predict the energies of interaction between graphene-based materials and molecular hydrogen has been investigated by Kocman et al. ${ }^{72}$ London dispersive forces can also be described using the random phase approximation (RPA) method, which accounts for electron-electron correlation effects from first principles. The RPA provides rather accurate predictions of surface adsorption behavior ${ }^{73-75}$ and bulk material properties. $^{76,77}$ However, it is very computationally demanding. Finally, the GW approximation ${ }^{78}$ has been used for accurate quasiparticle electronic band structure calculations. This manybody method corrects DFT using a self-energy operator consisting of Green's function (G) and the screened Coulomb interaction (W), and thereby inherently accounts for electron-electron correlation effects.

The height of the activation barrier to a given chemical modification of graphene can be related to the kinetics of the corresponding process using the Eyring equation. To accurately predict activation barriers, it is necessary to address the problem of the electron self-interaction error (SIE) in DFT exchange functionals. ${ }^{79}$ This can be achieved by admixing HF or exact exchange in DFT functionals. DFT functionals containing HF exchange are known as hybrid functionals. An ideal DFT method capable of accurately describing thermodynamics, kinetics and non-covalent interaction should thus be free of SIE and account for non-local electron correlation effects. This could potentially be achieved in various ways, for example by combining RPA with exact exchange. ${ }^{80,81}$ However, this would not be trivial to achieve, and careful testing of such approaches would be essential.

\subsection{Quantum Monte Carlo methods}

Quantum Monte Carlo (QMC) represents another strategy for solving the electronic Schrödinger equation from first principles. QMC methods are explicit many-body approaches based on the real-space random sampling of the electron configuration space. Two QMC methods are in common use, variational Monte Carlo (VMC) and diffusion Monte Carlo (DMC). The VMC method relies on the variational principle and stochastic integration of a quantum-mechanical total energy expectation value. Its main advantage is the ability to sample complicated wave functions including explicit correlation and to improve them variationally. A more powerful alternative to VMC is the fixed-node DMC method (FN-DMC), which relies on the projection (or enhancement) of the ground-state component from a given input trial electronic wave function in imaginary time. In combination with real-space sampling (that is a complete basis set, i.e. electrons can visit any point in the real space), FN-DMC provides exact solutions within the boundaries imposed by the fixed-node $\left(\Psi_{\mathrm{T}}=0\right)$ condition of the input trial state $\Psi_{\mathrm{T}}$. The fixed-node approximation is the one of multiple possible strategies for simulating Pauli exchange repulsion. FN-DMC thus efficiently accounts for electron-electron correlation effects from first principles. It should be noted that the QMC results are less sensitive to the one-electron basis sets used to construct trial wave functions since the electron correlations are simulated explicitly rather than by using many-body expansions in terms of one-particle states, as is the case in traditional wave function theory. QMC results have associated error bars that only converge slowly $(\propto 1 / \sqrt{ } K$ for calculations with $K$ independent sampling points), but the method's computational cost typically scales as a low-order polynomial (of order 3-4), which is significantly better than the scaling of CCSD(T)/CBS (of order 7) and thus enables studies of larger systems with comparable accuracy (as demonstrated in ref. 82). Moreover, QMC methods can be efficiently parallelized and implemented for both finite and periodic boundary conditions (Table 1). Consequently, they have great potential for use in electronic structure calculations on graphene and related compounds. In recent years, QMC methods have been used to study small conjugated hydrocarbons (benzene/ coronene) and their interactions with atoms/molecules ${ }^{72,82-86}$ and for explicit modelling of periodic graphene/graphite. ${ }^{84,87,88}$ For more details on QMC, we direct the reader to a pair of recent reviews (and references included therein). ${ }^{89,90}$

\subsection{Semiempirical methods}

Since the advent of quantum chemistry, there has been a continuous effort to develop fast electronic structure methods capable of treating large systems containing hundreds of atoms. One way of doing this is to introduce additional approximations to the HF method (Section 3.2) in the form of semiempirical parameters, which are derived by approximation or fitting to experimental results or data from higher-level calculations. Semiempirical methods such as AM1, ${ }^{91} \mathrm{PM} 3^{92}$ and $\mathrm{PM6}^{93}$ are very widely used in chemical research. In physics, the tight-binding (TB) semiempirical method is a 
similar approximate approach for predicting the electronic structure of periodic materials. ${ }^{94,95}$ In the TB approach, the wave function of a complex system is constructed as a superposition of the wave functions for isolated atoms located at the positions of the corresponding nuclei within the system of interest. It has been used successfully to describe graphene and its derivatives, ${ }^{96}$ achieving accuracies that rival higher level methods while enabling the simulation of systems comprising hundreds of atoms. For instance, ballistic transport in transistors based on the functionalized graphene ${ }^{97}$ were reported on the basis of the energy band calculation by high-level methods for graphane and graphone, subsequently fitted with a three-nearest neighbour $\mathrm{sp}^{3}$ tight-binding Hamiltonian. More recently, the TB approximation was used to study the electronic structures and optical properties of micrometer-scale partially and fully fluorinated graphene systems comprising $2400 \times 2400$ carbon atoms at GW accuracy. ${ }^{98}$ The TB approximation has also been generalized, leading to the development of density functional-based tight binding (DFTB). ${ }^{99}$ DFTB was subsequently improved by the incorporation of self-consistent redistribution of Mulliken charges (SCC-DFTB) ${ }^{100}$ to account for the Coulomb interaction between charge fluctuations, and by the addition of an empirical dispersion correction (SCC-DFTB-D). ${ }^{101}$ SCC-DFTB accounts for long-range electrostatic forces and self-interaction contributions, and has been used to investigate the correlation between the hydrogen superlattice structure on graphene and the band gap opening, ${ }^{102}$ and to explore the properties of graphene nanodots inside fluorographene. ${ }^{103}$

The approximations made in the creation of current semiempirical methods mean that they cannot accurately describe non-covalent interactions. This problem can be addressed by introducing empirical dispersion corrections (D) in the same way as was done for DFT in the creation of the DFT-D methods. In keeping with the established nomenclature, the suffix -D is appended to semiempirical methods corrected in this way, which include AM1-D and PM3-D. ${ }^{104}$ The latter of these two methods was successfully used to model the interactions of small molecules with aromatic systems ${ }^{105}$ and graphite. ${ }^{106}$ Hobza and coworkers developed the semiempirical method PM6-DH, which incorporates an additional correction term to describe hydrogen-bonding ${ }^{107}$ as a function of $\mathrm{H}$-bond length, donor- $\mathrm{H}$ - . acceptor angle and partial charges on the $\mathrm{H}$ and acceptor atoms. Additional variants of the $\mathrm{DH}$ correction, e.g., $\mathrm{DH}+{ }^{108}$ and $\mathrm{DH} 2,{ }^{109}$ which avoid double counting of the dispersion energy, are also available. These methods were used to model the adsorption of various molecules on graphene with quite good accuracy. ${ }^{110-112}$ A variant of the TB method incorporating an a posteriori dispersion correction has also been introduced, which performed well in the modelling of hydrogen physisorption on $\mathrm{PAH}$ and graphene and in predicting the bulk properties of graphite. ${ }^{113}$

\section{Empirical methods}

Whereas advanced quantum chemical methods provide highly accurate descriptions of systems comprising a few tens of

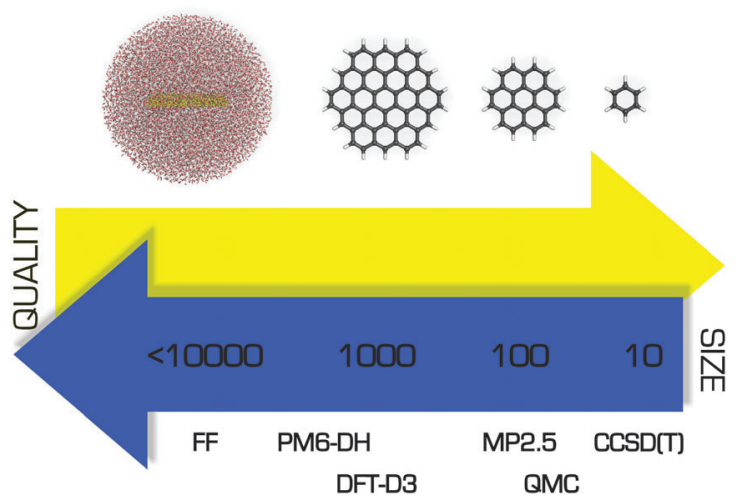

Fig. 5 Comparison of several theoretical approaches with respect to the size of the system that can be treated efficiently and the quality of the resulting description.

atoms, molecular mechanics (MM) can be used to perform calculations on systems comprising thousands of atoms (Fig. 5) such as nucleic acids, proteins, and nanostructures. Of course, this advantage is counterbalanced by many simplifications and limitations resulting from the omission of the electronic degrees of freedom: molecular mechanics only accounts for the motions of nuclei. In molecular mechanics, the system is considered to be an ensemble of beads and springs that are held together by simple harmonic forces. The core of the molecular mechanics calculation is a force field (also known as an empirical potential) consisting of a set of equations and some associated parameters that are used to describe the system's energetics. The resulting energy $E_{\mathrm{ff}}$ is calculated as the sum of several terms (eqn (1)) whose form and number is determined by the method's degree of simplification:

$$
E_{\mathrm{ff}}=E_{\text {bonded }}+E_{\mathrm{vdW}}+E_{\text {elec }}+\left(E_{\text {pol }}\right)+\left(E_{\text {other terms }}\right),
$$

here, $E_{\text {bonded }}$ represents the contributions to the total energy from bonding terms (bond stretching, angle bending, and torsion angle twisting), while $E_{\mathrm{vdw}}$ and $E_{\text {elec }}$ represent the non-bonding van der Waals and electrostatic terms, respectively. Further optional terms for polarization, $E_{\mathrm{pol}}$, and other additional energy terms (for instance dispersive many-body terms) are included in brackets. Non-covalent interactions are accounted for using simple expressions for the Coulombic (electrostatic) and van der Waals forces:

$$
\begin{gathered}
E_{\mathrm{vdW}}=4 \varepsilon_{i j}\left[\left(\frac{\sigma_{i j}}{r_{i j}}\right)^{12}-\left(\frac{\sigma_{i j}}{r_{i j}}\right)^{6}\right], \\
E_{\mathrm{elec}}=\frac{q_{i} q_{j}}{\varepsilon r_{i j}},
\end{gathered}
$$

Here, $\varepsilon_{i j}$ and $\sigma_{i j}$ are the Lennard-Jones (LJ) parameters, $r_{i j}$ is the interatomic distance, $\varepsilon$ is the relative permittivity and $q_{i}$ and $q_{j}$ are the partial electric charges. The first listed LJ parameter, $\varepsilon_{i j}$, specifies the well depth, which determines how strongly two particles interact; $\sigma_{i j}$ represents the distance at which the potential between the two particles is zero. The calculations can be performed with explicitly modelled solvent molecules, 
which are often essential when studying phenomena such as molecular recognition, protein folding, ${ }^{114}$ or liquid-phase exfoliation. ${ }^{115}$

\subsection{Current empirical force fields}

Numerous force fields for various kinds of structures have been developed over the past few decades. ${ }^{116-119}$ Force fields are often very specialized and designed to target quite narrow groups of molecules. The greatest number of empirical calculations are performed on biological systems and so efforts to develop and refine force fields have largely focused on proteins, nucleic acids, and so on. While the transferability of parameters from one molecule to another is one of the principal assumptions of molecular mechanics models, their validity is far from clear when transferring parameters from biomolecules to nanomaterials. Fortunately, several modified force field parameters have been developed specifically for simulating graphene. Table 2 compares the non-bonded parameters for aromatic carbon atoms from the three most widely used biomolecular force fields to those from several modified potentials that were developed for modelling carbon allotropes and which have been used by various groups. Since in most cases the carbon atoms in graphene are treated as uncharged LennardJones spheres, the molecular mechanics descriptions of the interactions between graphene and other molecules are governed exclusively by these non-bonded van der Waals parameters. Clearly, the listed force fields differ quite significantly with respect to these parameters, so it is important to choose a force field carefully if planning to use molecular mechanics to study graphene or its derivatives.

\subsection{Approximations employed by empirical force fields}

The advantage of the molecular mechanics approach over QM models is its simplicity and low computational cost (Fig. 5). Unfortunately, its approximations mean that many phenomena cannot be explicitly accounted for by the FFs. The problem of the quadrupole moment has already been mentioned, but there are other interactions that would be challenging or impossible to describe with a classical force field. These include the charge transport involved in many of graphene's intermolecular interactions, explicit polarization, and the charge redistribution caused by wrinkling of a graphene surface.

Table 2 Non-bonded parameters for aromatic carbon atoms from different force fields used in molecular dynamics simulations of graphene and graphene derivatives

\begin{tabular}{|c|c|c|}
\hline Force field & $\sigma[\AA]$ & $\varepsilon\left[\mathrm{kcal} \mathrm{mol}^{-1}\right]$ \\
\hline Parm $99^{116}$ & 3.39967 & 0.0860 \\
\hline OPLS $^{117}$ & 3.55000 & 0.0700 \\
\hline CHARMM $27^{118}$ & 3.55005 & 0.0700 \\
\hline Ulbricht et al. ${ }^{120}$ & 3.78108 & 0.0608 \\
\hline Girifalco et al. ${ }^{121}$ & 3.41214 & 0.0551 \\
\hline Cheng and Steele ${ }^{122}$ & 3.39967 & 0.0557 \\
\hline COMPASS $^{123 a}$ & 3.48787 & 0.0680 \\
\hline
\end{tabular}

${ }^{a}$ Uses 9-6 LJ potential.
The neglect of polarization interactions is perhaps the most serious deficiency of common pairwise additive force fields when modelling graphene and its derivatives. Conventional FFs treat electrostatic interactions using effective partial charges that are constructed to match electrostatic potentials obtained from QM calculations. The point charges are located on the atomic centres and are constant (i.e. conformation- and timeindependent). Consequently, it is impossible for the FF to react to changes in the molecular environment or to describe the way different solvents affect various interactions. In some force fields this problem is partly solved by adding an explicit term for electronic polarization. The contribution of polarization may be especially important in the case of nanomaterials, and it can be accounted for in several ways. A frequently used and technically simple option is the classical Drude model (the so-called "charge on spring" model), where an additional particle is attached to the atom. The particle has its own charge and, along with its attached atom, generates an induced dipole moment that depends on the external field. More detailed descriptions of the Drude model and its implementation can be found elsewhere. ${ }^{124}$ The Drude methodology was used by Ho et al. ${ }^{125}$ who studied the effect of graphene polarization on the structural properties of water molecules at a graphene-water interface. Their results suggested that the explicit inclusion of polarizability had no significant effects on the dynamics of the graphene-water system, and that the effect became even smaller for charged graphene. However, larger effects might be expected for ions and their arrangement near the graphene surface. A similar way of including polarizability is the rigid rod model. ${ }^{126}$ Like the Drude model, this approach involves attaching a virtual interaction site to the atom, but the assigned charge is kept at a fixed distance and is only permitted to rotate. The GRAPPA force field, which was specifically designed for simulations of water-graphitic interfaces, uses the rigid rod model. ${ }^{127} \mathrm{~A}$ third way of including polarization is to assign atomic polarizabilities to the atoms and then calculate the resulting induced dipoles, whose orientation is determined by the external field felt at each atomic site in the molecule. This approach was used by Schyman et al. ${ }^{128}$ in a study on the adsorption of water and ions on carbon surfaces including graphene, where the results obtained from polarizable and non-polarizable force fields were compared to quantum calculations. The authors suggested that the use of the polarizable force field substantially improved the description of graphenelike surfaces in the condensed phase.

Another drawback of current force fields is the pairwise additive approximation of the van der Waals interactions, where the resulting energy is calculated as a sum of contributions from individual pairs of atoms up to the cutoff distance. Many-body terms involving three or more atoms are not explicitly included. Although force fields are parameterized against experimental data and thus include many-body effects implicitly, in some cases it might be desirable to include at least three-body effects explicitly. In particular, many-body effects may be important for describing the behaviour of colloidal dispersions of nanomaterials or the intermolecular interactions of graphene sheets and nanotubes. ${ }^{57,129}$ 
Classical force fields do not allow bond cleavage and formation because they model bonds with harmonic potentials. This is sufficient for the study of various non-covalent modifications of graphene and other materials. However, a model capable of describing bond cleavage/formation is required for the study of any process involving chemical change such as chemisorption or chemical reactions. In such cases it is necessary to use methods that explicitly account for the system's electronic structure. Unfortunately, such methods can only be applied to relatively small model systems (Fig. 5). Empirical reactive force fields such as AIREBO, ${ }^{130}$ REBO, ${ }^{131}$ and ReaxFF ${ }^{132}$ were developed to enable the study of large reacting molecular systems. These force fields use the standard force field approximations but also include terms for bond formation and dissociation. A more detailed description of individual reactive force fields is beyond the scope of this review and can be found in the specialized literature. ${ }^{133,134}$

\section{Nuclear motion}

As electronic structure is within Born-Oppenheimer approximation solved separately and it was described in Sections 3 and 4, this section discusses methods that account for nuclear motion and can be used to estimate the associated physical-chemical quantities. Thermodynamic quantities (internal energy, enthalpy, entropy, etc.) for processes involving nuclear motion are typically obtained from molecular dynamics (MD) and Monte Carlo (MC) simulations that involve sampling configurational space. While the first method average time sequence of the required quantity, the latter collects values of the quantity corresponding to random configuration walk. ${ }^{135}$ Simulation methods that describe the studied system in terms of position and momentum vectors can be naturally extended to quantum versions (quantum MC and quantum MD) based on the nuclear wave function/density matrix as a central point.

\subsection{Molecular dynamics}

Molecular dynamics (MD) simulations usually use the laws of classical mechanics such as Newton's equations of motion to study the time evolution (dynamics) of a system:

$$
\boldsymbol{F}_{i}=m_{i} \frac{\mathrm{d}^{2}}{\mathrm{~d} t^{2}} \boldsymbol{r}_{i}(t), \quad i=1,2, \ldots, n .
$$

The force $\boldsymbol{F}_{i}$ acting on each atom $i$ (which has a mass $m_{i}$ and position $\boldsymbol{r}_{i}$ ) due to its interactions with other particles can be determined at any time $t$ during the simulation assuming that each atom's initial position and velocity is known. The force is enumerated as the negative gradient of the potential energy surface (PES)

$$
\boldsymbol{F}_{i}=-\nabla_{i} E\left(\boldsymbol{r}_{1}, \boldsymbol{r}_{2}, \ldots, \boldsymbol{r}_{n}\right) .
$$

Classical molecular dynamics is a method, which uses PES given as the predefined potential; either based on empirical data (force field) or on independent electronic structure calculations. The term $a b$ initio molecular dynamics (AIMD) ${ }^{136}$ is used if the electronic energy is acquired during the MD run.
AIMD has also been referred to as first principles MD, quantum chemical MD, on-the-fly MD, direct MD, potential-free MD and quantum MD.

Once the resulting force is known, new positions and velocities at time $t+\delta t$ are obtained by numerical solution of the equations. It is essential to select an appropriate time step $\delta t$. If a large time step is chosen the system may become unstable due to growing inaccuracies in the integration procedure. Time steps of 1-2 fs are typically used in classical MD simulations. This means that with current computer power it is possible to study dynamics on time scales of up to several microseconds. Perhaps the biggest benefit of this technique is its unique ability to provide information on the studied system at the atomistic level with femtosecond temporal resolutions. Moreover, specific techniques (for instance thermodynamic integration, potential of mean force, free energy perturbation, Jarzynski equality, etc. $)^{137}$ have been developed for use alongside MD to estimate the thermodynamic properties of the studied systems, making MD simulations potentially useful for investigating the thermodynamic changes accompanying the non-covalent functionalization of graphene.

Sometimes, it is not possible to neglect quantum effects associated with movements of atoms and molecules (see Section 6.8 for examples). In such cases it is necessary to work with a nuclear wave function known as a wavepacket in vibrational dynamics, which must be discretized and propagated. ${ }^{138}$ The system-bath approximation is typically used when simulating quantum objects on graphene. In this approximation, the quantum system is represented by a wavepacket and the initial classical surface is implemented in a way that accounts for lattice dynamics and corrugation. A recent study on the physisorption of atomic hydrogen on graphitic surfaces ${ }^{139}$ compared four different quantum mechanical techniques: close coupling wavepacket (CCWP) and reduced density matrix (RDM) propagation methods as well as the perturbation (PT) and effective Hamiltonian (EH) theories. All four methods' descriptions of hydrogen sticking were in reasonably good agreement. The CCWP and RDM methods described desorption well, but only the RDM method correctly captured the decay of the total trapped population. On the other hand, the PT and EH methods were around two orders of magnitude faster than CCWP and RDM. In the case of chemisorption, which involves stronger atom-surface coupling, perturbation methods cannot be accurate and CCWP or RDM should be used; ${ }^{140}$ the latter may be preferable because it can describe many phonon processes. An alternative approach to fully quantum problems based on Feynman's path integral from statistical quantum mechanics can also be formulated. Path integral molecular dynamics (PIMD) ${ }^{141}$ has been used successfully to study the adsorption of hydrogen on graphene and coronene. ${ }^{142}$

\subsection{Monte Carlo methods}

Monte Carlo methods are based on stochastic sampling, i.e. random walks ( $c f$. Section 3.4). Monte Carlo methods can be divided into methods which assume that classical mechanics is applicable (and energy is a continuous variable) and those 
which are based on the idea of discrete quantum energy levels. ${ }^{143}$ While the classical Monte Carlo (CMC) methods are less widely used than classical molecular dynamics in the modelling of graphene systems, quantum Monte Carlo (QMC) methods are commonly used to model strongly quantum interactions with graphene/graphite. The diffusion Monte Carlo (DMC) method is typically used to compute the ground vibrational state $(T=0 \mathrm{~K})$ of quantum systems on graphene. Thermodynamic properties at nonzero temperatures are computed using path-integral Monte Carlo (PIMC) methods, ${ }^{144}$ which directly sample the density matrix using the path integral approach and replace integrals with averages over samples, as is also done in PIMD.

\section{Selected applications}

\subsection{Interactions of graphenes}

Accurate descriptions of interactions with graphene are essential for understanding the structure and dynamics of graphenelike systems. The graphene-graphene interaction is of fundamental importance in many areas. Two graphene sheets can be stacked in a number of ways that differ in terms of the relative shifts of their basal planes. More attention is paid to the most stable AB-stacked arrangement, where half of the carbon atoms in the one layer sit directly above the centres of the hexagonal rings of the second layer. Nevertheless, determining the interlayer binding (cohesive) energy of graphene/graphite remains a significant challenge for theoreticians and experimentalists. ${ }^{145-149}$ Recently published benchmark data from thermal desorption spectroscopy suggested a value of $61 \mathrm{meV}$ per atom ${ }^{150}$ and prompted further in-depth theoretical investigations into the interlayer cohesive energy and vdW interactions in graphenelike systems. ${ }^{151-155} \mathrm{AB}$-stacked graphene is also an attractive object of study because it is potentially amenable to band gap tuning. ${ }^{156}$

Methods for modulating the band gap of graphene and its derivatives are highly desired because they make it possible to tune the material's electronic properties and could facilitate the design of a new generation of electronic devices. There are a number of ways in which the band gap of graphene could potentially be modified. One is to apply strain to the graphene. ${ }^{157,158}$ Alternatively, the adsorption of certain molecules on graphene induces symmetry breaking and hence band gap opening. ${ }^{159}$ It has been demonstrated that non-covalent functionalization of graphene with $\mathrm{Br}_{2}$ opens a relatively large band gap that can be further adjusted by using ultraviolet light to decompose the adsorbed $\mathrm{Br}_{2}$ molecules. ${ }^{160}$ A third option is the covalent modification of graphene. Fan et al. calculated that the electronic properties of graphene can be tuned by doping with either boron/nitrogen or joint $\mathrm{BN}$ domains. ${ }^{161}$ It was however shown that the chemical nature of $\mathrm{B} / \mathrm{N}$ dopants in graphene significantly changes the final doping effect (Fig. 6). ${ }^{162}$ It has also been suggested that the reaction of graphene with atomic hydrogen is able to reversibly (by annealing) convert this highly conductive species completely into graphane, which is an

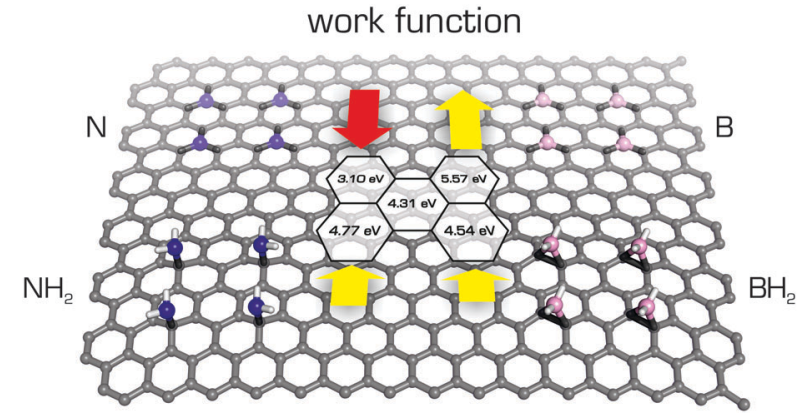

Fig. 6 The work functions $\left(W_{\mathrm{f}} \mathrm{s}\right)$ calculated using the PBEO functional of $\mathrm{B} / \mathrm{N}$-doped graphenes vary with the chemical nature of doping. ${ }^{162}$ The $W_{\mathrm{f}}$ value of pristine graphene $4.31 \mathrm{eV}$ (shown in the middle) increases in substitutionally B-doped graphene to $5.57 \mathrm{eV}$ and decreases to $3.10 \mathrm{eV}$ in substitutionally $\mathrm{N}$-doped graphene. On the other hand, the $W_{f}$ values increase in both graphenes with added $-\mathrm{NH}_{2}$ and $-\mathrm{BH}_{2}$ groups to 4.77 and $4.54 \mathrm{eV}$, respectively.

insulator. ${ }^{163}$ Moreover, Singh and co-workers ${ }^{164}$ interspaced small saturated graphene islands in the graphane host and showed that the energy gap of these islands is determined by their size. Specifically, DFT calculations indicated that smaller islands had larger energy gaps. Another way of engineering the band gap of graphene is to use graphene nanoribbons of different widths; the narrower the ribbon, the wider the gap. ${ }^{165,166}$ This approach could be particularly useful in printing processes. Graphene fluorination opens the band gap in a similar way to hydrogenation, ${ }^{167,168}$ and it has been suggested that the magnitude of the band gap could be tuned by adjusting the degree of fluorination ${ }^{169,170}$ or by replacing fluorine with heavier halogens. ${ }^{171}$

\subsection{Interactions of graphene with small molecules}

Graphene was quickly identified as a powerful adsorbent ${ }^{172}$ whose interactions with various molecules often induce specific physicochemical responses that could be exploited in new types of sensors. ${ }^{4,5,173}$ Moreover, non-covalent functionalization of the graphene surface substantially increases its potential range of applications. ${ }^{9}$ Therefore the interactions of graphene with small molecules have been studied extensively, both experimentally and computationally, in order to obtain information on the strength and nature of such interactions (for some examples see Fig. 7). Using DFT symmetry adapted perturbation theory (DFT-SAPT), ${ }^{174}$ which enables the decomposition of interaction energy into meaningful components, i.e., coulombic, polarization, dispersion terms etc., Lazar and coworkers showed that the adsorption of organic molecules was driven mostly by London dispersive forces. ${ }^{12}$ The same conclusion had previously been drawn in a study on the adsorption of water molecules to graphene. ${ }^{175}$ The adsorbates, which bind to graphene weakly via London dispersion forces, change its electronic structure only slightly but reduce the mobility of its electrons, ${ }^{176}$ which can be exploited in sensing applications. ${ }^{4}$ Recently, Zhou et al. ${ }^{177}$ studied the physisorption of benzene and benzene derivatives on graphene, and suggested that the benzene derivatives adsorb 

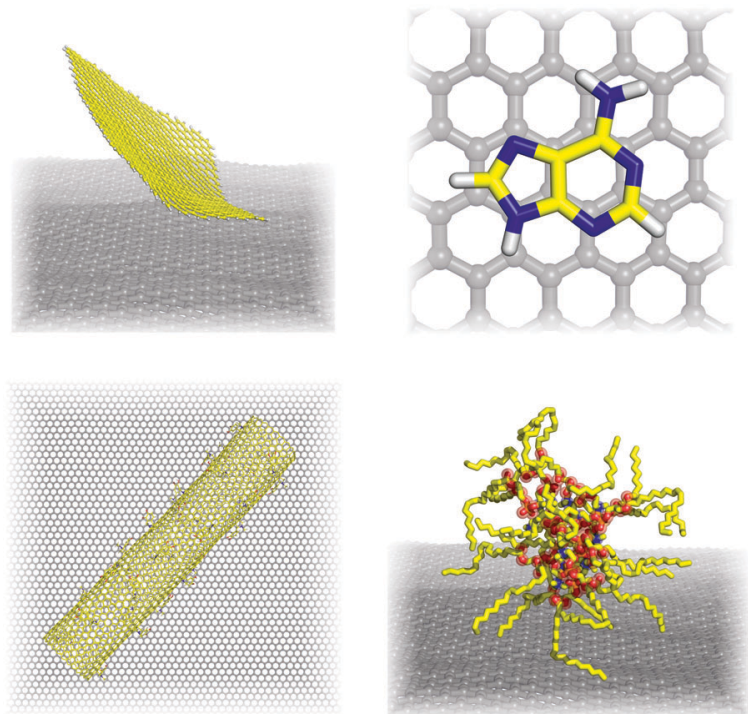

Fig. 7 Screenshots from molecular dynamics simulations of various processes taking place on a graphene surface: graphene exfoliation (top left), nucleobase adsorption (top right), graphene ...carbon nanotube assembly (bottom left), and the formation of a reverse lecithin micelle on a graphene surface.

more strongly than pure benzene regardless of their substituents' electronic properties.

Molecules adsorbed on graphene may also affect its electronic properties by donating (n-doping) or withdrawing (p-doping) electrons and thereby shifting its Fermi level. ${ }^{178,179}$ The same also applies for graphene supports. DFT calculations provide clear information about electron fluxes and can directly determine which adsorbates/supports donate/withdraw electrons to/ from graphene. This feature was also exploited to design graphene devices with a reasonably wide band gap, which can be used in graphene-based transistors. ${ }^{180,181}$ Such devices can be created from bilayer graphene sandwiched in between $\mathrm{FeCl}_{3}$ and $\mathrm{K}$ (Fig. 8). Calculations using the vdW-DF functional identified

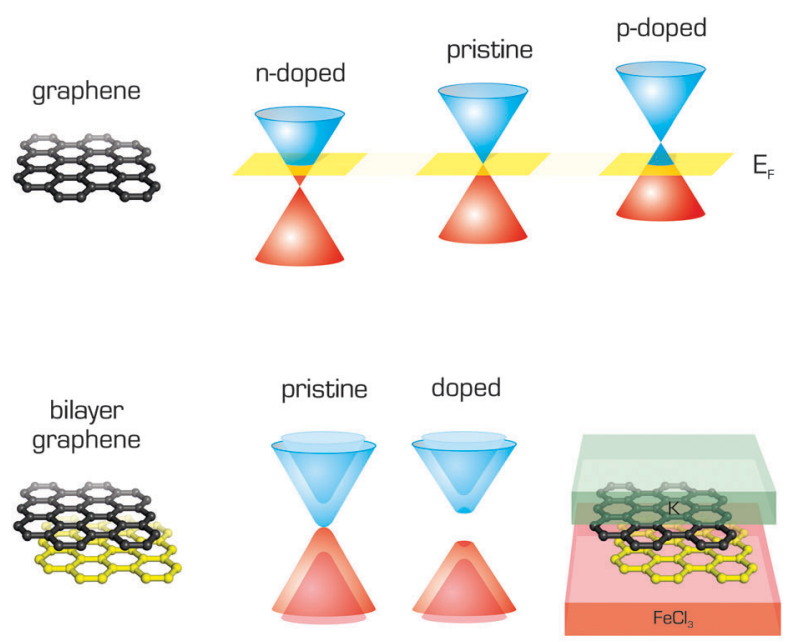

Fig. 8 Band structure of single layer graphene showing $p$ - and $n$-type doping with respect to the Fermi level, and band gap opening in bilayer graphene caused by doping.
$\mathrm{FeCl}_{3}$ as an electron acceptor capable of providing p-doped graphene and $\mathrm{K}$ as a donor providing $\mathrm{n}$-doped graphene. ${ }^{182}$

Many studies have investigated the binding energies of adsorbates to graphene using a very diverse portfolio of theoretical techniques. Unfortunately, the development of this field has been hampered by a lack of reliable experimental data, which makes it difficult to benchmark the performance of individual methods. Adsorption enthalpies are particularly suited for such comparisons because they correspond to welldefined processes, which can be modelled in a straightforward manner. Enthalpies are usually measured by temperature programmed desorption on highly oriented pyrolitic graphite (HOPG) ${ }^{183}$ or inverse gas chromatography on few-layered graphene. ${ }^{12,184}$ Calculations suggest that adsorption energies on single layer graphene are around $\sim 10 \%$ higher than those on few-layered graphene. ${ }^{184}$ The adsorption enthalpies derived from $a b$ initio MD simulations using the vdW-DF (optB88-vdW) functional were in good agreement with experimental data, suggesting that this non-local functional describes the binding energies of dispersion-bound molecules to graphene reasonably well. It is worth noting that force field simulations (using the OPLS-AA force field) also accurately predicted the relative binding enthalpies of the studied molecules, indicating that the same force field could be used to obtain preliminary estimates for the interaction energies of large molecules with graphene. If highly accurate predictions of binding energies of biomacromolecules to graphene are required, one should include contributions stemming from many-body terms. ${ }^{129}$

Preferred binding sites on the surface and energy differences between various binding sites can be estimated directly from theoretical calculations. Such information is important for understanding the friction on the graphene surface. Single atom adsorbates can bind at three sites (Fig. 9) referred to as on top (above the carbon atom perpendicular to the graphene sheet), on bond (above the carbon-carbon bond) and on hollow (above the centre of a "carbon hexagon"). Large molecules may have an even larger number of such high symmetry sites, as shown for tetracyanoethylene. ${ }^{185}$ Calculations can predict

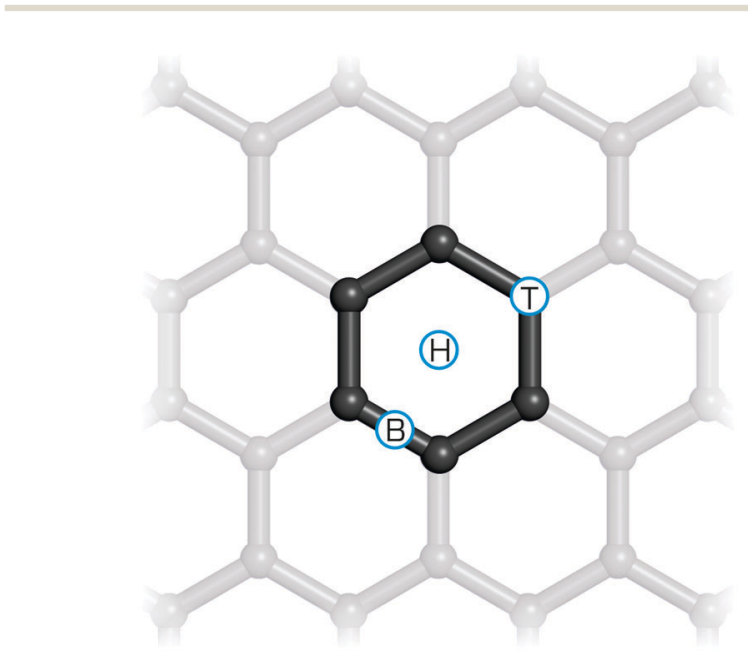

Fig. 9 On bond $(\mathrm{B})$, top $(\mathrm{T})$, and hollow $(\mathrm{H})$ adsorption sites on graphene. 
adsorption energies to individual sites and using the Boltzmann distribution law, the occupancy of individual sites can be estimated. Characterizing the potential energy surface of adsorbates sliding over the graphene increases the scope for understanding the friction that is generated. For example, calculations of this profile explained the contraintuitive increase in friction observed in a Pt atomic force microscopy tip moving over a graphene surface after fluorination. ${ }^{186}$

The strength of adsorption may depend not only on the adsorbate but also on its concentration and topology (the relative positions of individual adsorbates on the graphene surface). This indicates that the adsorbates significantly change the electronic structure of graphene and its binding involves some degree of covalent binding (chemisorption). The binding of fluorine or hydrogen atoms to graphene illustrates this phenomenon well. ${ }^{10}$ The bond dissociation energy of fluorine atoms at low concentration is only around $50 \mathrm{kcal} \mathrm{mol}{ }^{-1}$ whereas in fully fluorinated graphene (fluorographene or graphene fluoride) it is $112 \mathrm{kcal} \mathrm{mol}{ }^{-1} \cdot{ }^{187}$ The attachment of a fluorine atom to a carbon atom changes its $\mathrm{sp}^{2}$ hybridization state to $\mathrm{sp}^{3}$, inducing local structural buckling ( $c f$. Fig. 10). The degree of structural changes correlates with the strength of binding, which is reflected in the high resolution XPS spectrum of the corresponding atom. Consequently, high resolution XPS spectra can be used to decipher information about the binding of such atoms. ${ }^{188}$

The abovementioned information indicates that there is no sharp distinction between physisorption (non-covalent functionalization) and chemisorption (covalent functionalization) to graphene. In general, the interaction curve of a given adsorbate with graphene will feature two minima: one corresponding to physisorption (also known as the precursor state) and the other to chemisorption. ${ }^{140,142,189}$ These minima may be separated by an activation barrier (Fig. 10).
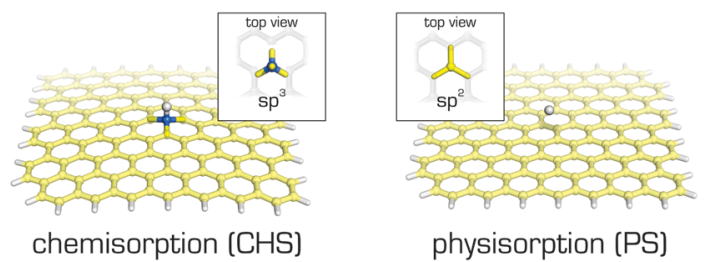

energy scale $[\mathrm{eV}]$

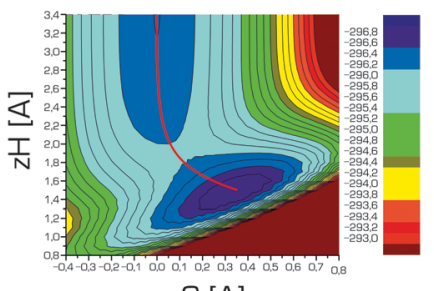

$\mathrm{zC}[\mathrm{A}]$

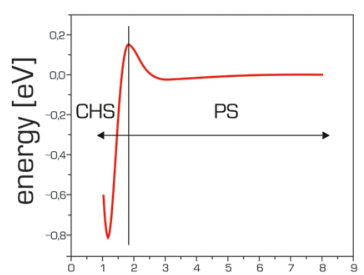

C-H distance [A]
Fig. 10 The potential energy surface (calculated using PBE-D2) for hydrogen adsorption on graphene features two separate energy minima corresponding to physisorbed (PS) and chemisorbed (CHS) complexes. $z \mathrm{H}$ and $z \mathrm{C}$ denote the $z$-coordinates of the hydrogen nucleus and the closest carbon atom, respectively.
Density functional theory and molecular dynamics were successfully used together to explore the adsorption of the amino acid leucine on graphene, ${ }^{190}$ revealing that under certain conditions leucine molecules adsorb spontaneously from solution. Moreover, it was suggested that the properties of the graphene could be tuned by controlling the orientation of the leucine molecules when they adsorbed. The adsorption of a somewhat larger tripeptide on graphene was studied by Camden et al. ${ }^{191}$ It was shown that the presence of water at the interface strongly influenced the peptide's binding and conformation, suggesting that the inclusion of explicit solvent molecules may be essential for a proper description of the properties of peptide systems on graphene. Furthermore, some organic molecules could form highly ordered self-assembled monolayers (SAM) and bilayers on the graphene surface. O'Mahony and coworkers ${ }^{192}$ used MD techniques to study the formation of alkylamine SAMs and the effect of different layer terminations on the adsorption of proteins on these platforms. It was suggested that alkylamine SAM assemblies could be used for instance for protein immobilization and exploited in targeted binding of specific molecules.

Molecular dynamics simulations appear to be useful for studying the wetting properties of graphene (and are widely used for this purpose), which are the subject of considerable ongoing debate. ${ }^{193,194}$ The surface tension of graphene should be measured on free standing graphene, which is still quite challenging to achieve experimentally because graphene is usually prepared on a support and may be contaminated by adsorbates from the atmosphere. ${ }^{195}$ On the other hand, such conditions are readily accessible in molecular simulations, which can estimate the contact angle on pure and free standing graphene. ${ }^{196}$ The hydrophobicity of graphene is crucial for many of its potential applications (in nanomedicine, sensing, filtration, surface coatings etc.) and depends on many variables such as the purity ${ }^{195}$ of the graphene sheet and the presence of defects $^{197,198}$ as well as the nature of the underlying support, whose wetting properties may affect (and be affected by) that of the graphene; this phenomenon is referred to as the wetting transparency of graphene. ${ }^{199,200} \mathrm{Li}$ and coworkers ${ }^{201}$ suggested that graphene and other graphitic surfaces may even be slightly hydrophilic due to the adsorption of hydrocarbons commonly present in the air. Detailed studies on this behaviour could lead to the design of novel functional devices. ${ }^{202}$

\subsection{Interactions of graphene with biomacromolecules}

A molecular-level understanding of nucleic acids' and proteins' conformational behaviour near graphene-like supports may be important in the design and optimization of new nanoscale devices. Such interactions could be important in nanomedicine, where graphene or its derivatives could act as enzymatic inhibitors, ${ }^{203}$ or in sensing since a variety of graphene-based sensors relying on different physicochemical principles have been proposed (Fig. 11). ${ }^{204}$ One should bear in mind that molecules proposed for sensing applications have to preserve their native structure upon adsorption to graphene to maintain their function. It was shown that it is theoretically feasible to construct 


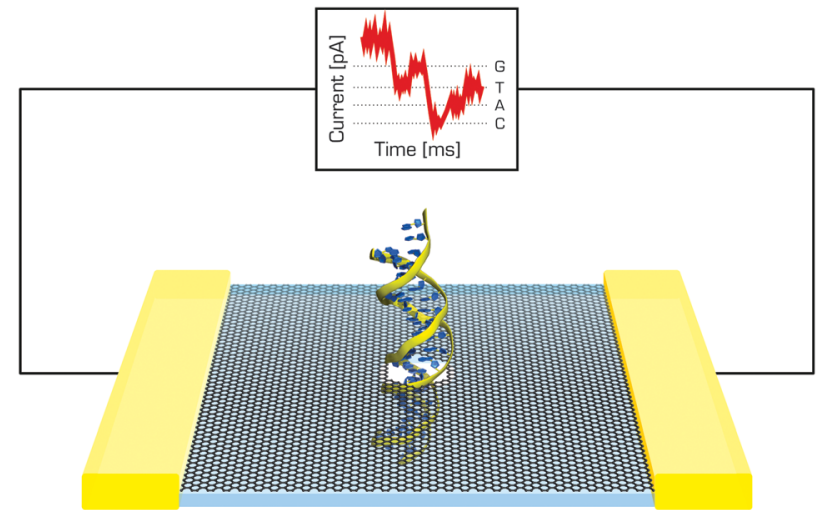

Fig. 11 DNA passing through the graphene nanopore may induce changes in the current, which could be used in DNA sequencing

very sensitive graphene devices for ssDNA sequencing as a rapid and cost-effective alternative to current techniques. ${ }^{205}$ Moreover, MD simulations of nucleic acid bases in solution suggest that graphene-base interactions are stronger than base-base stacking. ${ }^{206}$ It has also been observed that DNA bases interact strongly with graphene ${ }^{207-209}$ and that interactions with graphene can induce short DNA duplexes to partially unfold, mainly from the ends. $^{207}$ Such behaviour has also been reported for double stranded siRNA. ${ }^{210}$

\subsection{Graphene and metals}

The interactions of metals with graphene are very interesting, and complex. Naturally, graphene interacts with solid metals ${ }^{211}$ in electrical circuits, ${ }^{212}$ in graphene coated metals ${ }^{213}$ and during its synthesis by chemical vapour deposition. ${ }^{214-218}$ The interactions of metal nanoparticles with graphene are also very important because graphene provides a suitable platform on which to anchor such nanoparticles for catalytic, photocatalytic and sensor applications. ${ }^{219-223}$ Moreover, graphene is being considered as a potential replacement for the widely used graphite anodes of lithium (and more generally, alkali metal) ion batteries, because it is suggested to offer a higher lithium ion storage capacity and to reduce charging times. ${ }^{224,225}$ Numerous theoretical studies dealing with the adsorption and diffusion of alkali metal ions on pristine and functionalized graphene $\mathrm{e}^{226-229}$ as well as the positive influence of graphene defects on storage capacity ${ }^{225,230}$ can be found in the literature. Current progress in the use of graphene in energy applications and challenges for the field have been nicely summarized in recent reviews. ${ }^{231,232}$ Both individual metal atoms and small clusters may bond to graphene, altering its electronic and magnetic properties. ${ }^{227,233,234}$ It was suggested that graphene decorated with heavy adatoms could turn into a giant topological insulator, ${ }^{235}$ which might be used in magnetic storage devices. ${ }^{236,237}$ However, the correct description of magnetocrystalline anisotropy requires the usage of hybrid functionals ${ }^{238,239}$ and the inclusion of spin-orbit coupling. ${ }^{233}$ The nature of the metalgraphene interaction may be anywhere between non-covalent and partially covalent, ${ }^{240-242}$ indicating that any computational method used to study these interactions must reliably describe both London dispersive forces and chemical bonding. ${ }^{243,244}$ For catalytic applications involving bond-breaking and bond formation, it is also necessary to use methods that do not suffer from the electron self-interaction error, which leads to an underestimation of reaction barriers. Explicit relativistic effects ${ }^{245}$ should also be taken into account, especially when considering the interactions of heavy metals with graphene. Finally, when considering the interactions between metal adatoms and graphene, it is important to account for the spin-states of the metal and to be aware that these may change on binding. ${ }^{85}$

\subsection{Hybrid carbon systems}

The combination of graphene with other carbon allotropes such as nanotubes and fullerenes has opened up a new set of nanomaterials with many potential applications in areas such as printed electronics, conductive inks, reinforcement of polymers, etc. Computational modelling is playing an increasingly central role in studies on nanostructures because it enables the straightforward study of precisely defined structural motifs (joints) and because its atomistic resolution can help to elucidate unknown mechanisms and properties (Fig. 12). Interactions with fullerenes (mostly $\mathrm{C}_{60}$ ) are of particular interest. MD simulations have shown that fullerenes could potentially be used to detect defects on graphene. He et al. ${ }^{246}$ used $\mathrm{C}_{60}$ molecules to induce controlled ripples on the graphene sheet whose diffraction and interference can reveal cracks and defects on the surface. Several simulations of the diffusion of $\mathrm{C}_{60}$ molecules on graphene were performed at a constant temperature and with a temperature gradient. ${ }^{24,248}$ Moreover, Peng et al. ${ }^{249}$ suggested that $\mathrm{C}_{60}$ /graphene composites could be used for gas purification especially for some binary mixtures. Numerous computational studies on graphene-hybrid systems among other things are discussed in the recent review published by Zhang et al., which focuses primarily on the computational characterization and simulation of graphene-based materials. $^{250}$ It was recently demonstrated that it may be possible to combine graphene and carbon nanotubes in novel composite materials in which the graphene spontaneously rolls up around the nanotube or enters its interior. ${ }^{251-254}$ Graphene

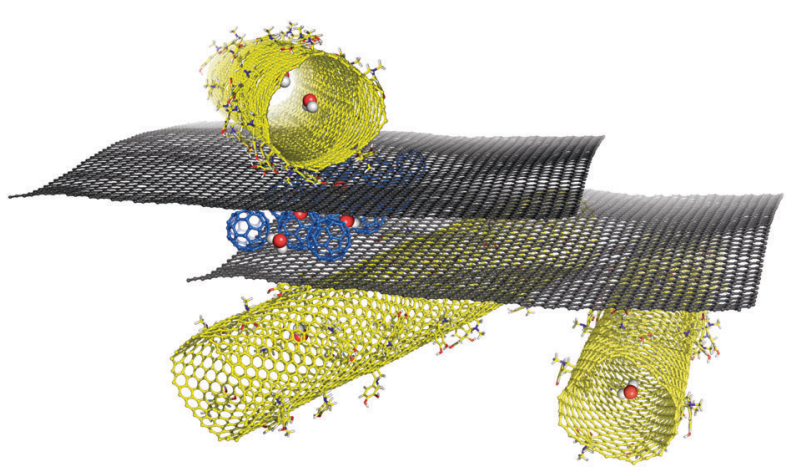

Fig. 12 Molecular modelling may provide unique molecular insight into the structures of graphene hybrid materials, which in turn may help us to design new functional nanosystems. 
can also interact with carbon nanotubes to form 3D pillared structures where individual graphene sheets are separated by perpendicularly oriented carbon nanotubes. MD techniques were used to study the mechanical and thermal properties of these nano-networks, ${ }^{255,256}$ and there is computational evidence that such pillared graphene structures could be used in gas separation $^{257}$ or hydrogen storage. ${ }^{258}$ Finally, Georgakilas et $a{ }^{259}$ dispersed graphene sheets in aquatic media using hydrophilic functionalized carbon nanotubes and produced highly conductive graphene ink. MD simulations suggested that the formation of aggregates from graphene and hydroxyphenyl-functionalized carbon nanotubes was kinetically controlled and led to a stable colloid dispersion.

\subsection{Graphene derivatives}

While the properties of pristine graphene have attracted great interest, modified graphene derivatives may be even more interesting, at least in certain applications. The derivative that has attracted most attention is graphene oxide (GO). One obstacle to the modelling of GO stems from its complex structure, which contains epoxy, hydroxyl and carboxy groups. Even the composition of GO is quite uncertain and may depend on the conditions applied in its preparation. ${ }^{260}$ Some models have been developed for studying the structure of GO, the most well-known and widely used of which is that of Lerf and Klinowski. ${ }^{261,262}$ This model suggests that alcohol and epoxy groups are distributed randomly on the basal plane while the carboxyl groups are located on the edges. The interactions of nucleobases and several amino acids with GO were studied computationally by Vovusha et al. ${ }^{263}$ It has been shown that complexes with GO are mainly stabilized by hydrogen bonding, in contrast with graphene complexes, which are stabilized mainly through dispersion interactions. Recently, Shih et al. used both experiments and molecular dynamics to study GO in solution ${ }^{264}$ and to analyse its aggregation as a function of the $\mathrm{pH}$ and the protonation of its functional groups. They observed that at low $\mathrm{pH}$ values, GO became less hydrophilic due to protonation and formed sandwich-like aggregates in which individual sheets were separated by a confined water layer. However, separate sheets were preferred at higher $\mathrm{pH}$ values. Other articles have examined the electrical, structural and chemical changes accompanying GO reduction, ${ }^{265,266}$ and a few recent atomistic works have investigated the effect of different reducing atmospheres on the reduction of GO, producing the results that complemented experimental investigations. ${ }^{267,268}$ In addition, two molecular dynamics studies investigated this material's unusual mechanical properties. ${ }^{194,269}$ Another interesting class of GO-based materials with diverse potential applications are the graphene oxide framework (GOF) materials. GOF is a porous material first synthesized in 2011 that consists of GO sheets connected by linkers. Nicolaï et al. developed molecular mechanics parameters for this material and used them to investigate its dynamic properties. ${ }^{270}$ They suggested that the density of linkers connecting the GO layers can be used to tune the diffusion properties of GOF materials.

Other graphene derivatives such as graphane and fluorographene have also been studied extensively by computational means. absorption spectra
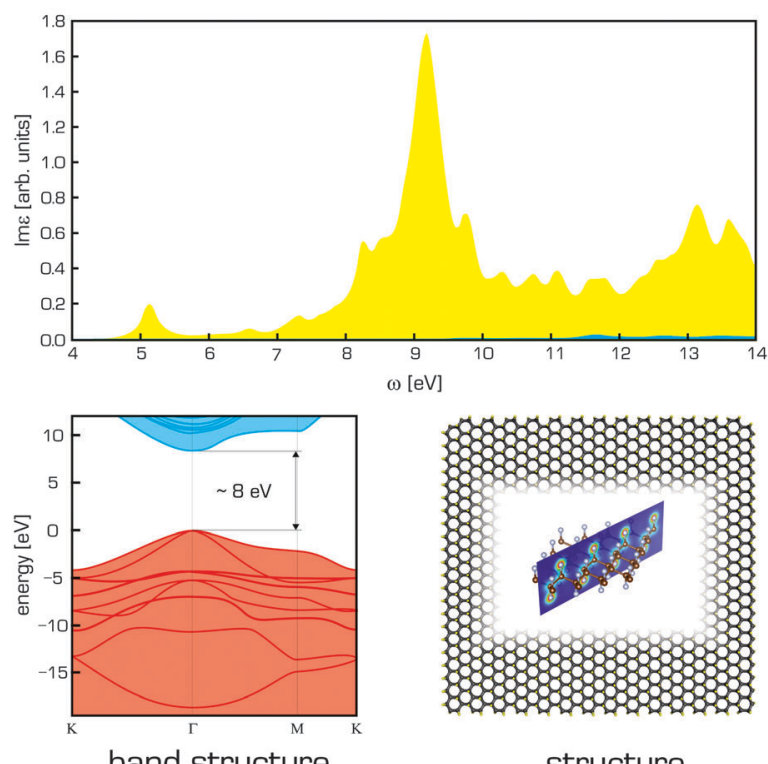

structure

Fig. 13 Structure of fluorographene is shown together with its electronic band structure (calculated using GW(PBE)) and BSE@GW(PBE) adsorption spectra for light polarization parallel (yellow) and perpendicular (blue) to the surface plane. ${ }^{276}$

Graphane was predicted as a graphene derivative on the basis of DFT calculations. ${ }^{271}$ Fluorographene and other graphene halides have been studied in some detail: ${ }^{272}$ different investigations have focused on their band gaps and optical transitions, ${ }^{273}$ the insulating properties of fluorographene $e^{167,274}$ and the broad UV/VIS photoluminescence band observed experimentally (Fig. 13). ${ }^{275} \mathrm{It}$ should however be noted that despite the use of computational methods that account for electron-electron and electron-hole correlation effects ${ }^{276,277}$ as well as the potential role of defects, ${ }^{98}$ it has not been possible to achieve satisfactory agreement between the computational results obtained to date and all of the available experimental data for fluorographene. Graphene-based materials have also been suggested for energy storage, fuel cells, and photovoltaic applications. The current state of computational chemistry methods for studying graphene-based energy materials is summarized in a review by Hughes et al. ${ }^{231}$ Furthermore, there is an intense effort led by the U.S. Department of Energy (DOE) to design novel materials for molecular storage (mainly molecular hydrogen) using graphene derivatives. Numerous computational studies have investigated the molecular interactions of hydrogen with pristine graphene and doped and substituted graphene materials with the aim of enhancing the physisorption of molecular hydrogen and increasing the adsorption capacity of these materials. $^{72,278-281}$

\subsection{Reactivity of graphene and graphene derivatives}

Computational studies can also provide unique insights into the mechanisms underpinning the chemical modification, i.e., reactivity, of graphene and its derivatives. For example, a study on cycloaddition reactions involving graphene predicted them 
to be thermodynamically favoured at edges whereas the surface was predicted to be unreactive. ${ }^{282,283}$ Very recently, fluorographene, which was once considered a nonreactive counterpart of Teflon, has been identified as a reactive material ${ }^{187,284}$ and a potential source of new graphene derivatives. ${ }^{189,285}$ Analyses of its mechanisms of reaction suggested that fully fluorinated graphene preferentially undergoes $\mathrm{S}_{\mathrm{N}} 2$-type substitutions. ${ }^{187}$ This finding poses new questions about the nature of the $\mathrm{C}-\mathrm{F}$ bonds in fluorographene and fluorinated graphenes. ${ }^{188}$ DFT calculations suggested that two fluorine atoms were inserted into graphene simultaneously during its reaction with $\mathrm{XeF}_{2}$. It was also shown that fluorination on one side facilitated the addition of another fluorine atom on the opposite side. ${ }^{286}$ Computations can also help to clarify the stability of graphene derivatives such as graphane, ${ }^{271}$ graphene halides $^{273}$ and graphene oxide. ${ }^{287}$ For example, although the structures and distributions of oxidized and unoxidized regions of GO are currently unclear, DFT studies conducted by Yang et $a .^{288}$ suggest that oxidation loci in GO are highly correlated, which is inconsistent with some previously proposed models that assume a random distribution of oxidized groups on GO.

\subsection{Graphene and quantum systems}

Finally, we comment on the delicate problems of very light and strongly quantum systems interacting with graphene and graphite. For such specific systems as $\mathrm{H}, \mathrm{H}_{2}$, and $\mathrm{He}$ as well as clusters, nanodroplets, films and layers of these substances, a full quantum treatment of both electrons and nuclei is often unavoidable. Tunnelling effects noticeably alter adsorption and diffusion barriers ${ }^{289}$ while nuclear delocalization effects change classical optimal geometrical structures and prohibit traditional approaches to computing the quantum zero-point energy. ${ }^{290,291}$

Most research efforts in this area have focused on the adsorption of hydrogen on graphene and graphite. A full quantum description of hydrogen and deuterium physisorption on graphite using an MP2 potential energy surface yielded sticking probabilities of the order of a few percent for collision energies of 0-25 meV. ${ }^{139,292,293}$ Sticking increased for collision energies close to those of the relevant diffraction resonances and was also enhanced by raising the surface temperature. Desorption time constants were in the range of 20-50 ps for a surface temperature of $300 \mathrm{~K}$. In contrast, graphene supported on a silicone oxide substrate or suspended over a hole in the substrate exhibited different physisorption properties. ${ }^{294}$ The sticking probabilities of hydrogen on these stabilized membranes at $10 \mathrm{~K}$ were high $(\sim 50 \%)$ at low collision energies $(\leq 10 \mathrm{meV})$, i.e. significantly larger than those for graphite. This was attributed to the different nature of the lattice vibrations in the two cases. More recently, the adsorption of hydrogen on graphene and graphite, ${ }^{140}$ and on graphene and coronene ${ }^{142}$ was studied by the wavepacket propagation method and path integral molecular dynamics. As both physisorption and chemisorption minima are present on the adsorption curve of hydrogen on graphene (Fig. 10), the barrier height between both minima contributes to the chemisorption probability. The barrier, which includes van der Waals, zero-point energy, quantum tunnelling and finite temperature effects, is approximately half or quarter of the height of the barrier predicted by DFT-GGA methods $(\sim 0.2 \mathrm{eV})$ for graphene. The overall chemisorption probability was about $20 \%$.

The adsorption of molecular hydrogen is often studied because of graphene's potential for hydrogen storage ( $c f$. Section 6.6). Kowalczyk et al. ${ }^{295}$ studied hydrogen in slit-like carbon nanopores at $77 \mathrm{~K}$ by grand canonical classical and path-integral Monte Carlo (PIMC) simulations. The volumetric density of stored energy in optimal carbon nanopores exceeded the DOE target for 2010 $\left(45 \mathrm{~kg} \mathrm{~m}^{-3}\right)$. For the narrow pores (pore width $H \in[0.59-0.7] \mathrm{nm}$ ), the reduction of the quantum isosteric enthalpy of adsorption at zero coverage was around $50 \%$ in comparison to the classical one and quantum confinement-inducing polymer shrinking was observed. Isosteric heats of adsorption for $\mathrm{H}_{2}, \mathrm{HD}$ and $\mathrm{D}_{2}$ as functions of coverage, and adsorption isotherms on graphite were computed by Wang and Johnson ${ }^{296}$ using the grand canonical classical PIMC method and shown to agree well with experimental results. The properties of $\mathrm{H}_{2}$ molecules adsorbed between graphite layers were also analysed by PIMD at temperatures of 300 to $900 \mathrm{~K}^{297}$ The storage capacities of carbon foams calculated by Yakobson et al. ${ }^{298}$ met material-based DOE targets
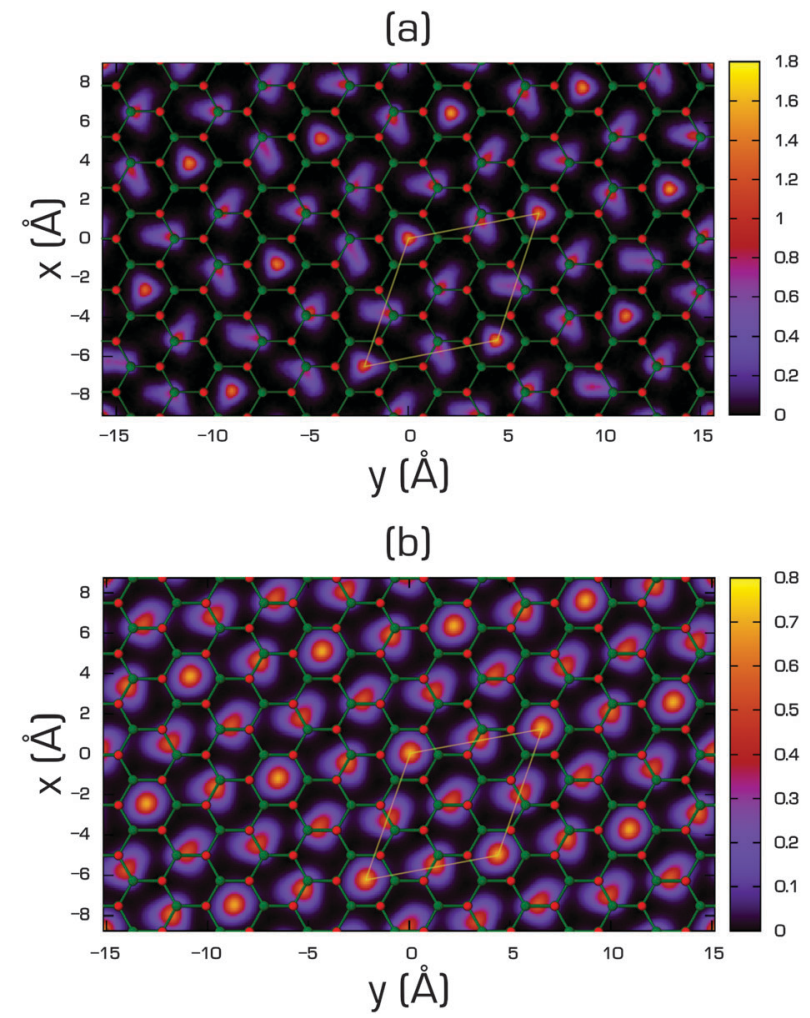

Fig. 14 Helium density (in $\AA^{-2}$ ) on the $x-y$ plane of the $2 / 7$ phase of ${ }^{4} \mathrm{He}$ on fluorographene (a) and on graphane (b) compared with the geometry of the substrate. Small red balls are centred on the position of fluorine/ hydrogen atoms and the small green ones on the carbon atoms. Thin white lines enclose the unit cell of the commensurate 2/7 phase [reprinted with permission from ref. 305. Copyright 2012 by the American Physical Society]. 
and are comparable to the capacities of a bundle of wellseparated open nanotubes of similar diameter. The authors also found that quantum effects appreciably changed the foams' adsorption properties and had to be taken into account. Recently, quantum effects and anharmonicity in the $\mathrm{H}_{2}-\mathrm{Li}^{+}$ benzene complex, a model for hydrogen storage materials, were studied $^{299}$ at zero temperature by DMC and rigid body DMC simulations at DFT PES. $\mathrm{H}_{2}$ molecules were delocalized above the $\mathrm{Li}^{+}$-benzene system and $\mathrm{H}_{2}$ binding enthalpy estimates were between 12.4-16.5 $\mathrm{kJ} \mathrm{mol}^{-1}$.

The importance of the substrate in understanding quantum films became evident with the detailed exploration of the phases of $\mathrm{He}$ and $\mathrm{H}_{2}$ on graphite, originating in the late 1960s (superfluidity, Bose-Einstein condensation, and idealized 2D bosonic gas are examples of fundamental phenomena of chemistry and physics). New phenomena occurring on the new 2D substrates have been envisaged opening new fundamental questions to address. While the phase behaviour of ${ }^{4} \mathrm{He}$ and para $-\mathrm{H}_{2}$ films (predicted by the PIMC method) on one side and both sides of graphene $e^{300,301}$ is expected to be similar to that on graphite, ${ }^{302-304}$ the behaviour predicted on fluorographene and graphane is different, due to different symmetry of the interaction potentials, doubled number of adsorption sites and larger corrugation for the adatom. ${ }^{305-307}$ For instance, the ground state of the He film on graphite is a $2 \mathrm{D}$ crystal commensurate with the substrate (the $\sqrt{ } 3 \times \sqrt{ } 3 R 30^{\circ}$ phase), while ${ }^{3} \mathrm{He}$ forms an anisotropic fluid and ${ }^{4} \mathrm{He}$ superfluid on fluorographene and graphane at the low coverage. ${ }^{305}$ At higher coverage values both the incommensurate triangular solid and the commensurate state at filling factor $2 / 7$ are found (Fig. 14). An interested reader may find more details on behaviour of monolayer quantum gases on graphene, graphane and fluorographene in the recent review (and references therein) by Reatto et $a .^{308}$

\section{Conclusions}

Computational chemistry provides valuable atomistic insights into the properties of systems that are relevant in biodisciplines and nanoscience. While computational methods are constantly evolving, they have already succeeded in several tasks and are undoubtedly becoming an integral part of the basic research toolkit. Because of the on-going increases in available computing power, the sizes of the systems amenable to modelling and the lengths of the simulation times that can be handled are both increasing, meaning that computational methods will continue to get more powerful and important. We have provided several examples showing how computational methods can be used to obtain insights into the physical and chemical properties of complex molecular systems related to graphene.

\section{Perspectives}

Despite all the great progress that has been made in modelling noncovalent interactions with graphene, many challenges remain to be addressed. There is still a need for a nonempirical theoretical method that reliably describes London dispersive forces without suffering from the electron-self interaction error and is also computationally affordable and easy to use. The recent progress in methods applying the adiabatic connection fluctuation-dissipation theorem is very promising in this respect. Robust testing of currently available methods is also highly desirable to assess their real performance. This task, is however, partially hampered by the lack of reliable experimental data addressing, e.g., the interaction energies between graphene and adsorbates.

One of the key issues that need to be addressed in today's empirical force fields is the explicit inclusion of polarization. This should be very important especially in describing adsorption processes involving graphene and its derivatives. Another challenge is the correct description of the long-range (asymptotic) dispersive interactions by empirical potentials. Whereas the classical $1 / R^{6}$ London formula results in a $1 / R^{4}$ distance dependence of the interaction energy for a molecule interacting with an infinite graphene sheet, the real distance dependence may be significantly different. ${ }^{309,310}$ Because some empirically corrected DFT methods (e.g. those based on the DFT-D approach) use this simple dispersion model, they may also describe the asymptotic interactions incorrectly. Unfortunately, the impact of this error is not currently well understood.

\section{Disclosure statement}

The authors declare that there is no conflict of interest regarding the publication of this paper.

\section{Acknowledgements}

The authors gratefully acknowledge project LO1305 of the Ministry of Education, Youths and Sports. MO acknowledges the Neuron fund to support science. We thank Matúš Dubecký, Piotr Błoński and Petr Lazar for helpful discussions.

\section{References}

1 K. S. Novoselov, A. K. Geim, S. V. Morozov, D. Jiang, Y. Zhang, S. V. Dubonos, I. V. Grigorieva and A. A. Firsov, Science, 2004, 306, 666-669.

2 A. K. Geim and K. S. Novoselov, Nat. Mater., 2007, 6, 183-191.

3 "Ten years in two dimensions." Editorial, Nat. Nanotechnol., 2014, 9, 725.

4 F. Schedin, A. K. Geim, S. V. Morozov, E. W. Hill, P. Blake, M. I. Katsnelson and K. S. Novoselov, Nat. Mater., 2007, 6, 652-655.

5 Y. Shao, J. Wang, H. Wu, J. Liu, I. A. Aksay and Y. Lin, Electroanalysis, 2010, 22, 1027-1036.

6 D. Rodrigo, O. Limaj, D. Janner, D. Etezadi, F. J. Garcia de Abajo, V. Pruneri and H. Altug, Science, 2015, 349, 165-168. 
7 F. Traversi, C. Raillon, S. M. Benameur, K. Liu, S. Khlybov, M. Tosun, D. Krasnozhon, A. Kis and A. Radenovic, Nat. Nanotechnol., 2013, 8, 939-945.

8 S. P. Koenig, L. Wang, J. Pellegrino and J. S. Bunch, Nat. Nanotechnol., 2012, 7, 728-732.

9 V. Georgakilas, M. Otyepka, A. B. Bourlinos, V. Chandra, N. Kim, K. C. Kemp, P. Hobza, R. Zbořil and K. S. Kim, Chem. Rev., 2012, 112, 6156-6214.

10 D. W. Boukhvalov and M. I. Katsnelson, J. Phys.: Condens. Matter, 2009, 21, 344205.

11 M. Rubeš, P. Nachtigall, J. Vondrášek and O. Bludský, J. Phys. Chem. C, 2009, 113, 8412-8419.

12 P. Lazar, F. Karlický, P. Jurečka, M. Kocman, E. Otyepková, K. Šafářová and M. Otyepka, J. Am. Chem. Soc., 2013, 135, 6372-6377.

13 R. Podeszwa, J. Chem. Phys., 2010, 132, 044704.

14 P. V. C. Medeiros, G. K. Gueorguiev and S. Stafström, Carbon, 2015, 81, 620-628.

15 Y. Zhao and D. G. Truhlar, J. Phys. Chem. C, 2008, 112, 4061-4067.

16 G. R. Jenness, O. Karalti and K. D. Jordan, Phys. Chem. Chem. Phys., 2010, 12, 6375-6381.

17 M. Kocman, M. Pykal and P. Jurečka, Phys. Chem. Chem. Phys., 2014, 16, 3144-3152.

18 S. Haldar, M. Kolář, R. Sedlák and P. Hobza, J. Phys. Chem. C, 2012, 116, 25328-25336.

19 Y. Hernandez, V. Nicolosi, M. Lotya, F. M. Blighe, Z. Sun, S. De, I. T. McGovern, B. Holland, M. Byrne, Y. K. Gun'Ko, J. J. Boland, P. Niraj, G. Duesberg, S. Krishnamurthy, R. Goodhue, J. Hutchison, V. Scardaci, A. C. Ferrari and J. N. Coleman, Nat. Nanotechnol., 2008, 3, 563-568.

20 C. Shih and S. Lin, J. Am. Chem. Soc., 2010, 132, 14638-14648.

21 S. Lin, C.-J. Shih, M. S. Strano and D. Blankschtein, J. Am. Chem. Soc., 2011, 133, 12810-12823.

22 Z. Qin, M. Taylor, M. Hwang, K. Bertoldi and M. J. Buehler, Nano Lett., 2014, 29, 7271-7282.

23 V. V. Gobre and A. Tkatchenko, Nat. Commun., 2013, 4, 2341.

24 M. Pykal, K. Šafářová, K. Machalová Šišková, P. Jurečka, A. B. Bourlinos, R. Zbořil and M. Otyepka, J. Phys. Chem. C, 2013, 117, 11800-11803.

25 F. London, Trans. Faraday Soc., 1937, 33, 8-26.

26 S. Grimme, J. Chem. Phys., 2003, 118, 9095.

27 R. A. Distasio Jr. and M. Head-Gordon, Mol. Phys., 2007, 105, 1073-1083.

28 T. Takatani, E. G. Hohenstein and C. D. Sherrill, J. Chem. Phys., 2008, 128, 124111.

29 M. Pitoňák, J. Řezáč and P. Hobza, Phys. Chem. Chem. Phys., 2010, 12, 9611-9614.

30 M. Pitoňák, P. Neogrády, J. Černý, S. Grimme and P. Hobza, ChemPhysChem, 2009, 10, 282-289.

31 C. Riplinger and F. Neese, J. Chem. Phys., 2013, 138, 034106.

32 C. Riplinger, B. Sandhoefer, A. Hansen and F. Neese, J. Chem. Phys., 2013, 139, 134101.
33 K. E. Riley, M. Pitonák, P. Jurecka and P. Hobza, Chem. Rev., 2010, 110, 5023-5063.

34 T. H. Dunning, J. Chem. Phys., 1989, 90, 1007.

35 C. J. Cramer, Essentials of Computational Chemistry: Theories and Models, John Wiley \& Sons, New York, 2nd edn, 2004.

36 A. Halkier, T. Helgaker, P. Jørgensen, W. Klopper, H. Koch, J. Olsen and A. K. Wilson, Chem. Phys. Lett., 1998, 286, 243-252.

37 P. L. Fast, M. L. Sanchez and D. G. Truhlar, J. Chem. Phys., 1999, 111, 2921-2926.

38 S. K. Min, E. C. Lee, H. M. Lee, D. Y. Kim, D. Y. Kim and K. S. Kim, J. Comput. Chem., 2008, 29, 1208-1221.

39 P. Jurečka and P. Hobza, J. Am. Chem. Soc., 2003, 125, 15608-15613.

40 J. Řezáč and P. Hobza, J. Chem. Theory Comput., 2013, 9, 2151-2155.

41 S. F. Boys and F. Bernardi, Mol. Phys., 1970, 19, 553-566.

42 M. Mentel and E. J. Baerends, J. Chem. Theory Comput., 2014, 10, 252-267.

43 I. Shin, M. Park, S. K. Min, E. C. Lee, S. B. Suh and K. S. Kim, J. Chem. Phys., 2006, 125, 234305.

44 C. Müller and B. Paulus, Phys. Chem. Chem. Phys., 2012, 14, 7605-7614.

45 A. Erba, S. Casassa, L. Maschio and C. Pisani, J. Phys. Chem. B, 2009, 113, 2347-2354.

46 C. Pisani, M. Busso, G. Capecchi, S. Casassa, R. Dovesi, L. Maschio, C. Zicovich-Wilson and M. Schütz, J. Chem. Phys., 2005, 122, 094113.

47 M. Marsman, A. Grüneis, J. Paier and G. Kresse, J. Chem. Phys., 2009, 130, 184103.

48 M. Del Ben, J. VandeVondele and B. Slater, J. Phys. Chem. Lett., 2014, 5, 4122-4128.

49 G. H. Booth, A. Grüneis, G. Kresse and A. Alavi, Nature, 2013, 493, 365-370.

50 S. Grimme, Wiley Interdiscip. Rev.: Comput. Mol. Sci., 2011, 1, 211-228.

51 S. Kristyán and P. Pulay, Chem. Phys. Lett., 1994, 229, 175-180.

52 P. Hobza, J. Šponer and T. Reschel, J. Comput. Chem., 1995, 16, 1315-1325.

53 P. Jurečka, J. Černý, P. Hobza and D. R. Salahub, J. Comput. Chem., 2007, 28, 555-569.

54 E. R. Johnson and A. D. Becke, J. Chem. Phys., 2005, 123, 024101.

55 S. Grimme, J. Comput. Chem., 2004, 25, 1463-1473.

56 S. Grimme, J. Comput. Chem., 2006, 27, 1787-1799.

57 S. Grimme, J. Antony, S. Ehrlich and H. Krieg, J. Chem. Phys., 2010, 132, 154104.

58 A. Tkatchenko and M. Scheffler, Phys. Rev. Lett., 2009, 102, 073005.

59 A. Tkatchenko, R. A. Distasio, R. Car and M. Scheffler, Phys. Rev. Lett., 2012, 108, 236402.

60 A. Tkatchenko, Adv. Funct. Mater., 2015, 25, 2054-2061.

61 S. Grimme, J. Chem. Phys., 2006, 124, 034108.

62 L. Goerigk and S. Grimme, J. Chem. Theory Comput., 2011, 7, 291-309. 
63 S. Kozuch, D. Gruzman and J. M. L. Martin, J. Phys. Chem. C, 2010, 114, 20801-20808.

64 T. Schwabe and S. Grimme, Phys. Chem. Chem. Phys., 2007, 9, 3397-3406.

65 M. Dion, H. Rydberg, E. Schröder, D. C. Langreth and B. I. Lundqvist, Phys. Rev. Lett., 2004, 92, 246401.

66 K. Lee, É. D. Murray, L. Kong, B. I. Lundqvist and D. C. Langreth, Phys. Rev. B: Condens. Matter Mater. Phys., 2010, 82, 081101.

67 J. Klimeš, D. R. Bowler and A. Michaelides, J. Phys.: Condens. Matter, 2010, 22, 022201.

68 O. A. Vydrov and T. van Voorhis, J. Chem. Phys., 2010, 133, 244103.

69 D. Langreth and J. Perdew, Phys. Rev. B: Condens. Matter Mater. Phys., 1977, 15, 2884-2901.

70 A. Tkatchenko, Adv. Funct. Mater., 2015, 25, 2054-2061.

71 Y. Zhao and D. G. Truhlar, Theor. Chem. Acc., 2008, 120, 215-241.

72 M. Kocman, P. Jurečka, M. Dubecký, M. Otyepka, Y. Cho and K. S. Kim, Phys. Chem. Chem. Phys., 2015, 17, 6423-6432.

73 J. Harl, L. Schimka and G. Kresse, Phys. Rev. B: Condens. Matter Mater. Phys., 2010, 81, 115126.

74 T. Olsen, J. Yan, J. J. Mortensen and K. S. Thygesen, Phys. Rev. Lett., 2011, 107, 156401.

75 F. Karlický, P. Lazar, M. Dubecký and M. Otyepka, J. Chem. Theory Comput., 2013, 9, 3670-3676.

76 H. Eshuis, J. E. Bates and F. Furche, Theor. Chem. Acc., 2012, 131, 1084.

77 X. Ren, P. Rinke, C. Joas and M. Scheffler, J. Mater. Sci., 2012, 47, 7447-7471.

78 L. Hedin, Phys. Rev., 1965, 139, A796.

79 A. J. Cohen, P. Mori-Sánchez and W. Yang, Chem. Rev., 2012, 112, 289-320.

80 A. Heßelmann and A. Görling, Mol. Phys., 2010, 108, 359-372.

81 A. Heßelmann and A. Görling, Phys. Rev. Lett., 2011, 106, 093001.

82 M. Dubecký, P. Jurečka, R. Derian, P. Hobza, M. Otyepka and L. Mitas, J. Chem. Theory Comput., 2013, 9, 4287-4292.

83 J. Ma, D. Alf, A. Michaelides and E. Wang, J. Chem. Phys., 2009, 130, 154303.

84 J. Ma, A. Michaelides and D. Alfè, J. Chem. Phys., 2011, 134, 134701.

85 J. Granatier, M. Dubecký, P. Lazar, M. Otyepka and P. Hobza, J. Chem. Theory Comput., 2013, 9, 1461-1468.

86 L. Horváthová, M. Dubecký, L. Mitas and I. Štich, Phys. Rev. Lett., 2012, 109, 053001.

87 P. Ganesh, J. Kim, C. Park, M. Yoon, F. A. Reboredo and P. R. C. Kent, J. Chem. Theory Comput., 2014, 10, 5318-5323.

88 H. Shin, S. Kang, J. Koo, H. Lee, J. Kim and Y. Kwon, J. Chem. Phys., 2014, 140, 114702.

89 W. M. C. Foulkes, L. Mitas, R. J. Needs and G. Rajagopal, Rev. Mod. Phys., 2001, 73, 33-83.

90 M. Dubecký, Acta Phys. Slovaca, 2014, 64, 501-574.
91 M. J. S. Dewar, E. G. Zoebisch, E. F. Healy and J. J. P. Stewart, J. Am. Chem. Soc., 1985, 107, 3902-3909.

92 J. J. P. Stewart, J. Comput. Chem., 1989, 10, 209-220.

93 J. J. P. Stewart, J. Mol. Model., 2007, 13, 1173-1213.

94 F. Bloch, Z. Phys. Chem., 1928, 52, 555-600.

95 J. C. Slater and G. F. Koster, Phys. Rev., 1954, 94, 1498-1524.

96 M. I. Katsnelson, Graphene: Carbon in Two Dimensions, Cambridge University Press, 1st edn, 2012.

97 G. Fiori, S. Lebègue, A. Betti, P. Michetti, M. Klintenberg, O. Eriksson and G. Iannaccone, Phys. Rev. B: Condens. Matter Mater. Phys., 2010, 82, 153404.

98 S. Yuan, M. Rösner, A. Schulz, T. O. Wehling and M. I. Katsnelson, Phys. Rev. Lett., 2015, 114, 047403.

99 D. Porezag, T. Frauenheim, T. Köhler, G. Seifert and R. Kaschner, Phys. Rev. B: Condens. Matter Mater. Phys., 1995, 51, 12947.

100 M. Elstner, D. Porezag, G. Jungnickel, J. Elsner, M. Haugk, T. Frauenheim, S. Suhai and G. Seifert, Phys. Rev. B: Condens. Matter Mater. Phys., 1998, 58, 7260.

101 M. Elstner, P. Hobza, T. Frauenheim, S. Suhai and E. Kaxiras, J. Chem. Phys., 2001, 114, 5149-5155.

102 R. Balog, B. Jørgensen, L. Nilsson, M. Andersen, E. Rienks, M. Bianchi, M. Fanetti, E. Laegsgaard, A. Baraldi, S. Lizzit, Z. Sljivancanin, F. Besenbacher, B. Hammer, T. G. Pedersen, P. Hofmann and L. Hornekaer, Nat. Mater., 2010, 9, 315-319.

103 M. A. Ribas, A. K. Singh, P. B. Sorokin and B. I. Yakobson, Nano Res., 2011, 4, 143-152.

104 J. P. McNamara and I. H. Hillier, Phys. Chem. Chem. Phys., 2007, 9, 2362-2370.

105 R. Sharma, J. P. McNamara, R. K. Raju, M. A. Vincent, I. H. Hillier and C. A. Morgado, Phys. Chem. Chem. Phys., 2008, 10, 2767-2774.

106 A. Ramraj and I. H. Hillier, J. Chem. Inf. Model., 2010, 50, 585-588.

107 J. Řezáč, J. Fanfrlík, D. Salahub and P. Hobza, J. Chem. Theory Comput., 2009, 5, 1749-1760.

108 M. Korth, J. Chem. Theory Comput., 2010, 6, 3808-3816.

109 M. Korth, M. Pitoňák, J. Řezáč and P. Hobza, J. Chem. Theory Comput., 2010, 6, 344-352.

110 E. G. Gordeev, M. V. Polynski and V. P. Ananikov, Phys. Chem. Chem. Phys., 2013, 15, 18815-18821.

111 M. A. Vincent and I. H. Hillier, J. Chem. Inf. Model., 2014, 54, 2255-2260.

112 S. Conti and M. Cecchini, J. Phys. Chem. C, 2015, 119, 1867-1879.

113 L. Zhechkov, T. Heine, S. Patchkovskii, G. Seifert and H. A. Duarte, J. Chem. Theory Comput., 2005, 1, 841-847.

114 Y. Levy and J. N. Onuchic, Annu. Rev. Biophys. Biomol. Struct., 2006, 35, 389-415.

115 J. N. Coleman, Adv. Funct. Mater., 2009, 19, 3680-3695.

116 J. Wang, P. Cieplak and P. A. Kollman, J. Comput. Chem., 2000, 21, 1049-1074.

117 W. L. Jorgensen, D. S. Maxwell and J. Tirado-Rives, J. Am. Chem. Soc., 1996, 118, 11225-11236. 
118 N. Foloppe and A. D. MacKerell, Jr., J. Comput. Chem., 2000, 21, 86-104.

119 C. Oostenbrink, A. Villa, A. E. Mark and W. F. van Gunsteren, J. Comput. Chem., 2004, 25, 1656-1676.

120 H. Ulbricht, G. Moos and T. Hertel, Phys. Rev. Lett., 2003, 90, 095501.

121 L. Girifalco, M. Hodak and R. Lee, Phys. Rev. B: Condens. Matter Mater. Phys., 2000, 62, 13104-13110.

122 A. Cheng and W. A. Steele, J. Chem. Phys., 1990, 92, 3867-3873.

123 H. Sun, J. Phys. Chem., 1998, 5647, 7338-7364.

124 V. M. Anisimov, I. V. Vorobyov, B. Roux and A. D. MacKerell, J. Chem. Theory Comput., 2007, 3, 1927-1946.

125 T. a. Ho and A. Striolo, J. Chem. Phys., 2013, 138, 054117.

126 F. Iori and S. Corni, J. Comput. Chem., 2008, 29, 1656-1666.

127 Z. E. Hughes, S. M. Tomásio and T. R. Walsh, Nanoscale, 2014, 6, 5438.

128 P. Schyman, W. L. Jorgensen and P. F. Field, J. Phys. Chem. Lett., 2013, 4, 468-474.

129 R. A. Distasio Jr., O. A. von Lilienfeld and A. Tkatchenko, Proc. Natl. Acad. Sci. U. S. A., 2012, 109, 14791-14795.

130 S. Stuart, A. Tutein and J. Harrison, J. Chem. Phys., 2000, 112, 6472-6486.

131 D. W. Brenner, O. A. Shenderova, J. A. Harrison, S. J. Stuart, B. Ni and S. B. Sinnott, J. Phys.: Condens. Matter, 2002, 14, 783-802.

132 A. C. T. van Duin, S. Dasgupta, F. Lorant and W. A. Goddard, J. Phys. Chem. A, 2001, 105, 9396-9409.

133 T. Liang, Y. K. Shin, Y.-T. Cheng, D. E. Yilmaz, K. G. Vishnu, O. Verners, C. Zou, S. R. Phillpot, S. B. Sinnott and A. C. T. van Duin, Annu. Rev. Mater. Res., 2013, 43, 109-129.

134 M. Z. S. Flores, P. A. S. Autreto, S. B. Legoas and D. S. Galvao, Nanotechnology, 2009, 20, 465704.

135 D. Frenkel and B. Smit, Understanding Molecular Simulation: From Algorithms to Applications, Elsevier, 2nd edn, 2001.

136 D. Marx and J. Hutter, NIC Series: Modern Methods and Algorithms of Quantum Chemistry, 2000, vol. 1, pp. 301-449.

137 W. F. van Gunsteren, X. Daura and A. E. Mark, Helv. Chim. Acta, 2002, 85, 3113-3129.

138 B. M. Garraway and K.-A. Suominen, Rep. Prog. Phys., 1995, 58, 365-419.

139 B. Lepetit, D. Lemoine, Z. Medina and B. Jackson, J. Chem. Phys., 2011, 134, 114705.

140 F. Karlický, B. Lepetit and D. Lemoine, J. Chem. Phys., 2014, 140, 124702.

141 M. E. Tuckerman, NIC Series: Quantum Simulations of Complex Many-Body Systems: From Theory to Algorithms, 2002, vol. 10, pp. 269-298.

142 E. R. M. Davidson, J. Klimeš, D. Alfè and A. Michaelides, ACS Nano, 2014, 8, 9905-9913.

143 M. Lewerenz, NIC Series: Quantum Simulations of Complex Many-Body Systems: From Theory to Algorithms, 2002, vol. 10, pp. 1-24.

144 B. Bernu and D. M. Ceperley, NIC Series: Quantum Simulations of Complex Many-Body Systems: From Theory to Algorithms, 2002, vol. 10, pp. 51-61.
145 L. A. Girifalco and R. A. Lad, J. Chem. Phys., 1956, 25, 693-697.

146 L. Benedict, N. Chopra and M. Cohen, Chem. Phys. Lett., 1998, 2614, 490-496.

147 N. L. Allinger, J. Am. Chem. Soc., 1977, 99, 8127-8134.

148 M. C. Schabel and J. L. Martins, Phys. Rev. B: Condens. Matter Mater. Phys., 1992, 46, 7185-7188.

149 J.-C. Charlier, X. Gonze and J.-P. Michenaud, Europhys. Lett., 1994, 28, 403-408.

150 R. Zacharia, H. Ulbricht and T. Hertel, Phys. Rev. B: Condens. Matter Mater. Phys., 2004, 69, 155406.

151 S. D. Chakarova-Käck, E. Schröder, B. I. Lundqvist and D. C. Langreth, Phys. Rev. Lett., 2006, 96, 146107.

152 S. Grimme, J. Phys. Chem. C, 2007, 111, 11199-11207.

153 L. Spanu, S. Sorella and G. Galli, Phys. Rev. Lett., 2009, 103, 196401.

154 S. Lebègue, J. Harl, T. Gould, J. G. Ángyán, G. Kresse and J. F. Dobson, Phys. Rev. Lett., 2010, 105, 196401.

155 X. Chen, F. Tian, C. Persson, W. Duan and N. Chen, Sci. Rep., 2013, 3, 3046.

156 J. B. Oostinga, H. B. Heersche, X. Liu, A. F. Morpurgo and L. M. K. Vandersypen, Nat. Mater., 2008, 7, 151-157.

157 Z. H. Ni, T. Yu, Y. H. Lu, Y. Y. Wang, Y. P. Feng and Z. X. Shen, ACS Nano, 2008, 2, 2301-2305.

158 G. Gui, J. Li and J. Zhong, Phys. Rev. B: Condens. Matter Mater. Phys., 2008, 78, 075435.

159 J. Berashevich and T. Chakraborty, Phys. Rev. B: Condens. Matter Mater. Phys., 2009, 80, 033404.

160 X. Fan, L. Liu, J. L. Kuo and Z. Shen, J. Phys. Chem. C, 2010, 114, 14939-14945.

161 X. Fan, Z. Shen, A. Q. Liu and J.-L. Kuo, Nanoscale, 2012, 4, 2157-2165.

162 P. Lazar, R. Zbořil, M. Pumera and M. Otyepka, Phys. Chem. Chem. Phys., 2014, 16, 14231-14235.

163 D. C. Elias, R. R. Nair, T. M. G. Mohiuddin, S. V. Morozov, P. Blake, M. P. Halsall, A. C. Ferrari, D. W. Boukhvalov, M. I. Katsnelson, A. K. Geim and K. S. Novoselov, Science, 2009, 323, 610-613.

164 A. K. Singh, E. S. Penev and B. I. Yakobson, ACS Nano, 2010, 4, 3510-3514.

165 L. Yang, C. H. Park, Y. W. Son, M. L. Cohen and S. G. Louie, Phys. Rev. Lett., 2007, 99, 186801.

166 M. Y. Han, B. Özyilmaz, Y. Zhang and P. Kim, Phys. Rev. Lett., 2007, 98, 206805.

167 R. Zbořil, F. Karlický, A. B. Bourlinos, T. A. Steriotis, A. K. Stubos, V. Georgakilas, K. Šafářová, D. Jančík, C. Trapalis and M. Otyepka, Small, 2010, 6, 2885-2891.

168 D. K. Samarakoon, Z. Chen, C. Nicolas and X.-Q. Wang, Small, 2011, 7, 965-969.

169 J. T. Robinson, J. S. Burgess, C. E. Junkermeier, S. C. Badescu, T. L. Reinecke, F. K. Perkins, M. K. Zalalutdniov, J. W. Baldwin, J. C. Culbertson, P. E. Sheehan and E. S. Snow, Nano Lett., 2010, 10, 3001-3005.

170 Z. Wang, J. Wang, Z. Li, P. Gong, X. Liu, L. Zhang, J. Ren, H. Wang and S. Yang, Carbon, 2012, 50, 5403-5410. 
171 F. Karlický, R. Zbořil and M. Otyepka, J. Chem. Phys., 2012, 137, 034709.

172 J. Wang, Z. Chen and B. Chen, Environ. Sci. Technol., 2014, 48, 4817-4825.

173 Y. Liu, X. Dong and P. Chen, Chem. Soc. Rev., 2012, 41, 2283-2307.

174 K. Szalewicz, Wiley Interdiscip. Rev.: Comput. Mol. Sci., 2012, 2, 254-272.

175 G. R. Jenness and K. D. Jordan, J. Phys. Chem. C, 2009, 113, 10242-10248.

176 L. Kong, A. Enders, T. S. Rahman and P. A. Dowben, J. Phys.: Condens. Matter, 2014, 26, 443001.

177 P.-P. Zhou and R.-Q. Zhang, Phys. Chem. Chem. Phys., 2015, 17, 12185-12193.

178 S. K. Mali, J. Greenwood, J. Adisoejoso, R. Phillipson and S. De Feyter, Nanoscale, 2015, 7, 1566-1585.

179 T. Hu and I. C. Gerber, J. Phys. Chem. C, 2013, 117, 2411-2420.

180 F. Schwierz, Nat. Nanotechnol., 2010, 5, 487-496.

181 T. Ohta, A. Bostwick, T. Seyller, K. Horn and E. Rotenberg, Science, 2006, 313, 951-954.

182 J. W. Yang, G. Lee, J. S. Kim and K. S. Kim, J. Phys. Chem. Lett., 2011, 2, 2577-2581.

183 H. Ulbricht, R. Zacharia, N. Cindir and T. Hertel, Carbon, 2006, 44, 2931-2942.

184 P. Lazar, E. Otyepková, P. Banáš, A. Fargašová, K. Šafářová, L. Lapčík, J. Pechoušek, R. Zbořil and M. Otyepka, Carbon, 2014, 73, 448-453.

185 Y. H. Lu, W. Chen, Y. P. Feng and P. M. He, J. Phys. Chem. $B, 2009,113,2-5$.

186 Q. Li, X.-Z. Liu, S.-P. Kim, V. B. Shenoy, P. E. Sheehan, J. T. Robinson and R. W. Carpick, Nano Lett., 2014, 14, 5212-5217.

187 M. Dubecký, E. Otyepková, P. Lazar, F. Karlický, M. Petr, K. Čépe, P. Banáš, R. Zbořil and M. Otyepka, J. Phys. Chem. Lett., 2015, 6, 1430-1434.

188 S. Zhou, S. D. Sherpa, D. W. Hess and A. Bongiorno, J. Phys. Chem. C, 2014, 118, 26402-26408.

189 P. Lazar, C. K. Chua, K. Holá, R. Zbořil, M. Otyepka and M. Pumera, Small, 2015, 11, 3790-3796.

190 W. Qin, X. Li, W.-W. Bian, X.-J. Fan and J.-Y. Qi, Biomaterials, 2010, 31, 1007-1016.

191 A. N. Camden, S. a. Barr and R. J. Berry, J. Phys. Chem. B, 2013, 117, 10691-10697.

192 S. O'Mahony, C. O’Dwyer, C. A. Nijhuis, J. C. Greer, A. J. Quinn and D. Thompson, Langmuir, 2013, 29, 7271-7282.

193 H. Zhou, P. Ganesh, V. Presser, M. C. F. Wander, P. Fenter, P. R. C. Kent, D. E. Jiang, A. A. Chialvo, J. McDonough, K. L. Shuford and Y. Gogotsi, Phys. Rev. B: Condens. Matter Mater. Phys., 2012, 85, 035406.

194 N. Wei, C. Lv and Z. Xu, Langmuir, 2014, 30, 3572-3578.

195 K. Xu and J. R. Heath, Nat. Mater., 2013, 12, 872-873.

196 F. Taherian, V. Marcon, N. F. A. van der Vegt and F. Leroy, Langmuir, 2013, 29, 1457-1465.

197 R. Raj, S. C. Maroo and E. N. Wang, Nano Lett., 2013, 13, 1509-1515.
198 C.-J. Shih, M. S. Strano and D. Blankschtein, Nat. Mater., 2013, 12, 866-869.

199 J. Rafiee, X. Mi, H. Gullapalli, A. V. Thomas, F. Yavari, Y. Shi, P. M. Ajayan and N. A. Koratkar, Nat. Mater., 2012, 11, 217-222.

200 C.-J. J. Shih, Q. H. Wang, S. Lin, K.-C. C. Park, Z. Jin, M. S. Strano and D. Blankschtein, Phys. Rev. Lett., 2012, 109, 176101.

201 Z. Li, Y. Wang, A. Kozbial, G. Shenoy, F. Zhou, R. McGinley, P. Ireland, B. Morganstein, A. Kunkel, S. P. Surwade, L. Li and H. Liu, Nat. Mater., 2013, 12, 925-931.

202 N. Patra, B. Wang and P. Král, Nano Lett., 2009, 9, 3766-3771.

203 X. Sun, Z. Feng, T. Hou and Y. Li, ACS Appl. Mater. Interfaces, 2014, 6, 7153-7163.

204 J. Li, Y. Zhang, J. Yang, K. Bi, Z. Ni, D. Li and Y. Chen, Phys. Rev. E: Stat., Nonlinear, Soft Matter Phys., 2013, 87, 062707.

205 S. K. Min, W. Y. Kim, Y. Cho and K. S. Kim, Nat. Nanotechnol., 2011, 6, 162-165.

206 V. Spiwok, P. Hobza and J. Řezáč, J. Phys. Chem. C, 2011, 115, 19455-19462.

207 X. Zhao, J. Phys. Chem. C, 2011, 115, 6181-6189.

208 M. Kabeláč, O. Kroutil, M. Předota, F. Lankaš and M. Šíp, Phys. Chem. Chem. Phys., 2012, 14, 4217-4229.

209 Y. Cho, S. K. Min, J. Yun, W. Y. Kim, A. Tkatchenko and K. S. Kim, J. Chem. Theory Comput., 2013, 9, 2090-2096.

210 S. Mogurampelly, S. Panigrahi, D. Bhattacharyya, A. K. Sood and P. K. Maiti, J. Chem. Phys., 2012, 137, 054903.

211 J. Wintterlin and M. L. Bocquet, Surf. Sci., 2009, 603, 1841-1852.

212 G. Giovannetti, P. A. Khomyakov, G. Brocks, V. M. Karpan, J. van Den Brink and P. J. Kelly, Phys. Rev. Lett., 2008, 101, 026803.

213 L. Hu, H. S. Kim, J. Y. Lee, P. Peumans and Y. Cui, ACS Nano, 2010, 4, 2955-2963.

214 P. W. Sutter, J.-I. Flege and E. A. Sutter, Nat. Mater., 2008, 7, 406-411.

215 X. Li, W. Cai, L. Colombo and R. S. Ruoff, Nano Lett., 2009, 9, 4268-4272.

216 Z. Sun, Z. Yan, J. Yao, E. Beitler, Y. Zhu and J. M. Tour, Nature, 2010, 468, 549-552.

217 Y. Zhang, L. Zhang and C. Zhou, Acc. Chem. Res., 2013, 46, 2329-2339.

218 P. Lacovig, M. Pozzo, D. Alfè, P. Vilmercati, A. Baraldi and S. Lizzit, Phys. Rev. Lett., 2009, 103, 166101.

219 R. Kou, Y. Shao, D. Mei, Z. Nie, D. Wang, C. Wang, V. V. Viswanathan, S. Park, I. A. Aksay, Y. Lin, Y. Wang and J. Liu, J. Am. Chem. Soc., 2011, 133, 2541-2547.

220 C. Xu, X. Wang and J. Zhu, J. Phys. Chem. C, 2008, 112, 19841-19845.

221 R. Muszynski, B. Seger and P. V. Kamat, J. Phys. Chem. C, 2008, 112, 5263-5266.

222 P. V. Kamat, J. Phys. Chem. Lett., 2010, 1, 520-527.

223 S. Guo, D. Wen, Y. Zhai, S. Dong and E. Wang, ACS Nano, 2010, 4, 3959-3968. 
224 J. Liu, Y. H. Xue, M. Zhang and L. M. Dai, MRS Bull., 2012, 37, 1265-1272.

225 X. Fan, W. T. Zheng and J. L. Kuo, ACS Appl. Mater. Interfaces, 2012, 4, 2432-2438.

226 M. Khantha, N. A. Cordero, L. M. Molina, J. A. Alonso and L. A. Girifalco, Phys. Rev. B: Condens. Matter Mater. Phys, 2004, 70, 1-8.

227 K. T. Chan, J. B. Neaton and M. L. Cohen, Phys. Rev. B: Condens. Matter Mater. Phys., 2008, 77, 235430.

228 E. Lee and K. A. Persson, Nano Lett., 2012, 12, 4624-4628. 229 R. P. Hardikar, D. Das, S. S. Han, K. Lee and A. K. Singh, Phys. Chem. Chem. Phys., 2014, 16, 16502-16508.

230 L.-J. Zhou, Z. F. Hou and L.-M. Wu, J. Phys. Chem. C, 2012, 116, 21780-21787.

231 Z. E. Hughes and T. R. Walsh, Nanoscale, 2015, 7, 6883-6908.

232 R. Raccichini, A. Varzi, S. Passerini and B. Scrosati, Nat. Mater., 2014, 14, 271-279.

233 P. Lazar, J. Granatier, J. Klimeš, P. Hobza and M. Otyepka, Phys. Chem. Chem. Phys., 2014, 16, 20818-20827.

234 P. V. C. Medeiros, G. K. Gueorguiev and S. Stafström, Phys. Rev. B: Condens. Matter Mater. Phys., 2012, 85, 205423.

$235 \mathrm{~J} . \mathrm{Hu}, \mathrm{J}$. Alicea, R. Wu and M. Franz, Phys. Rev. Lett., 2012, 109, 266801.

236 A. Ehrlicher and J. H. Hartwig, Nat. Mater., 2011, 10, 12-13.

237 I. G. Rau, S. Baumann, S. Rusponi, F. Donati, S. Stepanow, L. Gragnaniello, J. Dreiser, C. Piamonteze, F. Nolting, S. Gangopadhyay, O. R. Albertini, R. M. Macfarlane, C. P. Lutz, B. A. Jones, P. Gambardella, A. J. Heinrich and H. Brune, Science, 2014, 344, 988-992.

238 P. Błoński and J. Hafner, Phys. Rev. B: Condens. Matter Mater. Phys., 2009, 79, 224418.

239 I. Beljakov, V. Meded, F. Symalla, K. Fink, S. Shallcross, M. Ruben and W. Wenzel, Nano Lett., 2014, 14, 3364-3368.

240 P. A. Khomyakov, G. Giovannetti, P. C. Rusu, G. Brocks, J. van Den Brink and P. J. Kelly, Phys. Rev. B: Condens. Matter Mater. Phys., 2009, 79, 195425.

241 J. Granatier, P. Lazar, M. Otyepka and P. Hobza, J. Chem. Theory Comput., 2011, 7, 3743-3755.

242 M. Stella, S. J. Bennie and F. R. Manby, Mol. Phys., 2015, $1-7$.

243 T. P. Hardcastle, C. R. Seabourne, R. Zan, R. M. D. Brydson, U. Bangert, Q. M. Ramasse, K. S. Novoselov and A. J. Scott, Phys. Rev. B: Condens. Matter Mater. Phys., 2013, 87, 195430.

244 T. Olsen and K. S. Thygesen, Phys. Rev. B: Condens. Matter Mater. Phys., 2013, 87, 075111.

245 M. Iliaš, V. Kellö and M. Urban, Acta Phys. Slovaca, 2010, 60, 259-391.

246 Y. Z. He, H. Li, P. C. Si, Y. F. Li, H. Q. Yu, X. Q. Zhang, F. Ding, K. M. Liew and X. F. Liu, Appl. Phys. Lett., 2011, 98, 2012-2015.

247 M. Neek-Amal, N. Abedpour, S. N. Rasuli, A. Naji and M. R. Ejtehadi, Phys. Rev. E: Stat., Nonlinear, Soft Matter Phys., 2010, 82, 051605.

248 a. Lohrasebi, M. Neek-Amal and M. R. Ejtehadi, Phys. Rev. E: Stat., Nonlinear, Soft Matter Phys., 2011, 83, 042601.
249 X. Peng, D. Cao and W. Wang, Ind. Eng. Chem. Res., 2010, 49, 8787-8796.

250 T. Zhang, Q. Xue, S. Zhang and M. Dong, Nano Today, 2012, 7, 180-200.

251 D. Xia, J. Xie, H. Chen, C. Lv, F. Besenbacher, Q. Xue and M. Dong, Small, 2010, 6, 2010-2019.

252 Z. Zhang and T. Li, Appl. Phys. Lett., 2010, 97, 2012-2015.

253 Y. Jiang, H. Li, Y. Li, H. Yu, K. M. Liew, Y. He and X. Liu, ACS Nano, 2011, 5, 2126-2133.

254 N. Patra, Y. Song and P. Král, ACS Nano, 2011, 5, 1798-1804.

255 V. Varshney, S. S. Patnaik, A. K. Roy, G. Froudakis and B. L. Farmer, ACS Nano, 2010, 4, 1153-1161.

256 L. Xu, N. Wei, Y. Zheng, Z. Fan, H.-Q. Wang and J.-C. Zheng, J. Mater. Chem., 2012, 22, 1435-1444.

257 R. P. Wesołowski and A. P. Terzyk, Phys. Chem. Chem. Phys., 2011, 13, 17027-17029.

258 G. K. Dimitrakakis, E. Tylianakis and G. E. Froudakis, Nano Lett., 2008, 8, 3166-3170.

259 V. Georgakilas, A. Demeslis, E. Ntararas, A. Kouloumpis, K. Dimos, D. Gournis, M. Kocman, M. Otyepka and R. Zbořil, Adv. Funct. Mater., 2015, 25, 1481-1487.

260 J. G. S. Moo, B. Khezri, R. D. Webster and M. Pumera, ChemPhysChem, 2014, 15, 2922-2929.

261 H. He, J. Klinowski, M. Forster and A. Lerf, Chem. Phys. Lett., 1998, 287, 53-56.

262 A. Lerf, H. He, M. Forster and J. Klinowski, J. Phys. Chem. B, 1998, 102, 4477-4482.

263 H. Vovusha, S. Sanyal and B. Sanyal, J. Phys. Chem. Lett., 2013, 4, 3710-3718.

264 C.-J. Shih, S. Lin, R. Sharma, M. S. Strano and D. Blankschtein, Langmuir, 2012, 28, 235-241.

265 C. Mattevi, G. Eda, S. Agnoli, S. Miller, K. A. Mkhoyan, O. Celik, D. Mastrogiovanni, C. Cranozzi, E. Carfunkel and M. Chhowalla, Adv. Funct. Mater., 2009, 19, 2577-2583.

266 A. Bagri, C. Mattevi, M. Acik, Y. J. Chabal, M. Chhowalla and V. B. Shenoy, Nat. Chem., 2010, 2, 581-587.

267 R. M. Abolfath and K. Cho, J. Phys. Chem. A, 2012, 116, 1820-1827.

268 B. Narayanan, S. L. Weeks, B. N. Jariwala, B. Macco, J.-W. Weber, S. J. Rathi, M. C. M. van de Sanden, P. Sutter, S. Agarwal and C. V. Ciobanu, J. Vac. Sci. Technol., A, 2013, 31, 040601.

269 J. Zhang and D. Jiang, Carbon, 2014, 67, 784-791.

270 A. Nicolaï, P. Zhu, B. G. Sumpter and V. Meunier, J. Chem. Theory Comput., 2013, 9, 4890-4900.

271 J. O. Sofo, A. S. Chaudhari and G. D. Barber, Phys. Rev. B: Condens. Matter Mater. Phys., 2007, 75, 153401.

272 F. Karlický, K. Kumara Ramanatha Datta, M. Otyepka and R. Zbořil, ACS Nano, 2013, 7, 6434-6464.

273 F. Karlický, R. Zbořil and M. Otyepka, J. Chem. Phys., 2012, 137, 034709.

274 R. R. Nair, W. Ren, R. Jalil, I. Riaz, V. G. Kravets, L. Britnell, P. Blake, F. Schedin, A. S. Mayorov, S. Yuan, M. I. Katsnelson, H. M. Cheng, W. Strupinski, L. G. Bulusheva, A. V. Okotrub, I. V. Grigorieva, 
A. N. Grigorenko, K. S. Novoselov and A. K. Geim, Small, 2010, 6, 2877-2884.

275 K. J. Jeon, Z. Lee, E. Pollak, L. Moreschini, A. Bostwick, C. M. Park, R. Mendelsberg, V. Radmilovic, R. Kostecki, T. J. Richardson and E. Rotenberg, ACS Nano, 2011, 5, 1042-1046.

276 F. Karlický and M. Otyepka, J. Chem. Theory Comput., 2013, 9, 4155-4164.

277 F. Karlický and M. Otyepka, Ann. Phys., 2014, 526, 408-414.

278 S. Patchkovskii, J. S. Tse, S. N. Yurchenko, L. Zhechkov, T. Heine and G. Seifert, Proc. Natl. Acad. Sci. U. S. A., 2005, 102, 10439-10444.

279 A. Du, Z. Zhu and S. C. Smith, J. Am. Chem. Soc., 2010, 132, 2876-2877.

280 K. C. Kemp, H. Seema, M. Saleh, N. H. Le, K. Mahesh, V. Chandra and K. S. Kim, Nanoscale, 2013, 5, 3149-3171.

281 M. Seydou, K. Lassoued, F. Tielens, F. Maurel, F. Raouafi and B. Diawara, RSC Adv., 2015, 5, 14400-14406.

282 Y. Cao, S. Osuna, Y. Liang, R. C. Haddon and K. N. Houk, J. Am. Chem. Soc., 2013, 135, 17643-17649.

283 I. K. Petrushenko, Monatsh. Chem., 2014, 145, 891-896.

284 K. E. Whitener, R. Stine, J. T. Robinson and P. E. Sheehan, J. Phys. Chem. C, 2015, 119, 10507-10512.

285 V. Urbanová, K. Holá, A. B. Bourlinos, K. Čépe, A. Ambrosi, A. H. Loo, M. Pumera, F. Karlický, M. Otyepka and R. Zbořil, Adv. Mater., 2015, 27, 2305-2310.

286 S. S. Lee, S. W. Jang, K. Park, E. C. Jang, J. Y. Kim, D. Neuhauser and S. Lee, J. Phys. Chem. C, 2013, 117, 5407-5415.

287 S. Zhou and A. Bongiorno, Sci. Rep., 2013, 3, 2484.

288 J. Yang, G. Shi, Y. Tu and H. Fang, Angew. Chem., Int. Ed., 2014, 53, 10190-10194.

289 E. M. McIntosh, K. T. Wikfeldt, J. Ellis, A. Michaelides and W. Allison, J. Phys. Chem. Lett., 2013, 4, 1565-1569.

290 P. Svrčková, A. Vítek, F. Karlický, I. Paidarová and R. Kalus, J. Chem. Phys., 2011, 134, 224310.

291 F. Calvo, F. Y. Naumkin and D. J. Wales, J. Chem. Phys., 2011, 135, 124308.
292 Z. Medina and B. Jackson, J. Chem. Phys., 2008, 128, 114704.

293 V. Buch, J. Chem. Phys., 1989, 91, 4974.

294 B. Lepetit and B. Jackson, Phys. Rev. Lett., 2011, 107, 236102.

295 P. Kowalczyk, P. A. Gauden, A. P. Terzyk and S. K. Bhatia, Langmuir, 2007, 23, 3666-3672.

296 Q. Wang and J. K. Johnson, Mol. Phys., 1998, 95, 299-309.

297 C. P. Herrero and R. Ramírez, Phys. Rev. B: Condens. Matter Mater. Phys., 2010, 82, 174117.

298 A. K. Singh, J. Lu, R. S. Aga and B. I. Yakobson, J. Phys. Chem. C, 2011, 115, 2476-2482.

299 S. J. Kolmann, J. H. D’Arcy and M. J. T. Jordan, J. Chem. Phys., 2013, 139, 234305.

300 Y. Kwon and D. M. Ceperley, Phys. Rev. B: Condens. Matter Mater. Phys., 2012, 85, 224501.

301 M. C. Gordillo, Phys. Rev. B: Condens. Matter Mater. Phys., 2013, 88, 10-13.

302 F. F. Abraham and J. Q. Broughton, Phys. Rev. Lett., 1987, 59, 64-67.

303 M. Pierce and E. Manousakis, Phys. Rev. B: Condens. Matter Mater. Phys., 1999, 59, 3802-3814.

304 D. Sato, K. Naruse, T. Matsui and H. Fukuyama, Phys. Rev. Lett., 2012, 109, 235306.

305 M. Nava, D. E. Galli, M. W. Cole and L. Reatto, Phys. Rev. B: Condens. Matter Mater. Phys., 2012, 86, 174509.

306 L. Reatto, M. Nava, D. E. Galli, C. Billman, J. O. Sofo and M. V. Cole, J. Phys.: Conf. Ser., 2012, 400, 012010.

307 M. Nava, D. E. Galli, M. W. Cole and L. Reatto, J. Low Temp. Phys., 2013, 171, 699-710.

308 L. Reatto, D. E. Galli, M. Nava and M. W. Cole, J. Phys.: Condens. Matter, 2013, 25, 443001.

309 J. F. Dobson, A. White and A. Rubio, Phys. Rev. Lett., 2006, 96, 073201.

310 T. Gould, E. Gray and J. F. Dobson, Phys. Rev. B: Condens. Matter Mater. Phys., 2009, 79, 113402. 